\title{
The role of magnetic handedness in magnetic cloud propagation
}

\author{
U. Taubenschuss ${ }^{1,6}$, N. V. Erkaev $^{3,4}$, H. K. Biernat ${ }^{1,2}$, C. J. Farrugia ${ }^{5}$, C. Möstl ${ }^{1,2}$, and U. V. Amerstorfer ${ }^{1}$ \\ ${ }^{1}$ Space Research Institute, Austrian Academy of Sciences, 8042 Graz, Austria \\ ${ }^{2}$ Institute of Physics, University of Graz, 8010 Graz, Austria \\ ${ }^{3}$ Institute of Computational Modelling, 660036 Krasnoyarsk, Russia \\ ${ }^{4}$ Siberian Federal University, 660041 Krasnoyarsk, Russia \\ ${ }^{5}$ Space Science Center and Department of Physics, University of New Hampshire, Durham, NH 03824, USA \\ ${ }^{6}$ Department of Physics and Astronomy, University of Iowa, Iowa City, IA, USA
}

Received: 2 November 2009 - Revised: 19 April 2010 - Accepted: 4 May 2010 - Published: 7 May 2010

\begin{abstract}
We investigate the propagation of magnetic clouds (MCs) through the inner heliosphere using 2.5-D ideal magnetohydrodynamic (MHD) simulations. A numerical solution is obtained on a spherical grid, either in a meridional plane or in an equatorial plane, by using a Roe-type approximate Riemann solver in the frame of a finite volume approach. The structured background solar wind is simulated for a solar activity minimum phase. In the frame of MC propagation, special emphasis is placed on the role of the initial magnetic handedness of the MC's force-free magnetic field because this parameter strongly influences the efficiency of magnetic reconnection between the MC's magnetic field and the interplanetary magnetic field. Magnetic clouds with an axis oriented perpendicular to the equatorial plane develop into an elliptic shape, and the ellipse drifts into azimuthal direction. A new feature seen in our simulations is an additional tilt of the ellipse with respect to the direction of propagation as a direct consequence of magnetic reconnection. During propagation in a meridional plane, the initial circular cross section develops a concave-outward shape. Depending on the initial handedness, the cloud's magnetic field may reconnect along its backside flanks to the ambient interplanetary magnetic field (IMF), thereby losing magnetic flux to the IMF. Such a process in combination with a structured ambient solar wind has never been analyzed in detail before. Furthermore, we address the topics of force-free magnetic field conservation and the development of equatorward flows ahead of a concave-outward shaped MC. Detailed profiles are presented for the radial evolution of magnetoplasma and geometrical parameters. The principal features seen in our MHD simulations are in good agreement with in-situ mea-
\end{abstract}

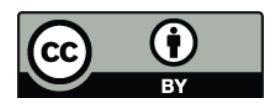

Correspondence to: U. Taubenschuss (ulrich.taubenschuss@oeaw.ac.at) surements performed by spacecraft. The 2.5-D studies presented here may serve as a basis under more simple geometrical conditions to understand more complicated effects seen in 3-D simulations.

Keywords. Interplanetary physics (Interplanetary magnetic fields; Solar wind plasma) - Space plasma physics (Numerical simulation studies)

\section{Introduction}

The term magnetic cloud was introduced to describe a subset of interplanetary coronal mass ejections (ICMEs) having a set of well-defined properties (Burlaga et al., 1981). Numerous detections of magnetic cloud signatures in spacecraft data reveal the following characteristic features. Inside the $\mathrm{MC}$, the plasma pressure is lower than in the ambient solar wind but the total pressure, i.e., plasma pressure plus magnetic pressure, is enhanced. This indicates a strong magnetic field (approximately $15-30 \mathrm{nT}$ at $1 \mathrm{AU}$ ), which additionally executes a smooth rotation while measured by spacecraft moving through the cloud structure (Burlaga et al., 1981; Lepping et al., 1990). The mass density and the ion temperature are lower inside the magnetic cloud when compared to the ambient solar wind (Gosling et al., 1973; Richardson and Cane, 1995). Furthermore, MC plasmas show a highly variable abundance of Helium (0-20\%) which exceeds the Helium abundance in the slow solar wind ( $\sim \%)$ (Borrini et al., 1982). The fact that these signatures are observed for only 30-40\% of ICMEs (Gosling, 1990) may be strongly linked to an observational selection effect because ICME measurements are very sensitive with regard to the trajectory of the spacecraft through the structure. Furthermore, interactions between two different ICMEs or between ICMEs and other

Published by Copernicus Publications on behalf of the European Geosciences Union. 
slow or fast solar wind streams may extinguish some of these properties (Richardson and Cane, 2004; Lugaz et al., 2008; Dasso et al., 2009).

According to the large amount of data acquired by various spacecraft, MCs can be visualized as large-scale magnetic flux ropes. These flux ropes emerge as CMEs from the Sun's surface and propagate into interplanetary space at speeds usually higher than that of the ambient solar wind. During propagation, magnetic clouds are expanding. A typical diameter at a heliospheric distance of $1 \mathrm{AU}$ is in the range 0.2-0.4 AU (Klein and Burlaga, 1982; Hu and Sonnerup, 2002). A bidirectional flow of supra-thermal electrons along magnetic field lines inside the cloud supports the assumption that magnetic clouds are closed loops with their feet still attached to the Sun's atmosphere (Bame et al., 1981; Gosling et al., 1987; Kahler and Reames, 1991; Farrugia et al., 1993). In contrast to this picture, Vandas et al. (1991) and Vandas et al. (1993) developed a model for magnetic clouds with spheroidal topology, the so-called spheromak model.

Magnetic field observations of MCs are often analyzed using a minimum variance analysis (Bothmer and Schwenn, 1998) or by fitting observations to a force-free magnetic field model of constant $\alpha$ with cylindrical symmetry (Lundquist, 1950; Burlaga, 1988). On the basis of the force-free field model, additional information on important MC parameters can be deduced, such as the orientation of the flux rope's axis, the magnetic field strength on the axis and the cloud's diameter. Empirical laws for the cloud's geometrical parameters and several other physical quantities as a function of distance from the Sun can be found in Bothmer and Schwenn (1998), Wang et al. (2005), Liu et al. (2005, 2006) and Leitner et al. (2007).

Results for flux rope fitting to spacecraft observations are improved by taking into account a strong deformation of the MC cross section due to interaction with a structured ambient solar wind (Mulligan and Russell, 2001; Hidalgo, 2003; Démoulin and Dasso, 2009). One step further in flux rope fitting is to work with non-force-free models, e.g., to fit parameters on the basis of a magnetohydrostatic model as done in a Grad-Shafranov reconstruction approach (Hau and Sonnerup, 1999; Hu and Sonnerup, 2002; Möstl et al., 2008). This self-consistent technique has the advantage that the cloud's shape does not have to be assumed prior to the analysis. However, certain aspects of the propagation of MCs and their interaction with a structured ambient solar wind require full magnetohydrodynamic simulations which address not only kinematic (Riley and Crooker, 2004; Owens, 2006) and hydrodynamic aspects (Gosling et al., 1998; Odstrčil and Pizzo, 1999; Cargill et al., 2000) but emphasize also the important role of the magnetic field. Recent efforts concentrate on merging of models from different heliospheric domains trying to capture the initiation of a CME near the Sun as well as covering its propagation from the solar corona to the orbit of Earth and beyond (Odstrcil et al., 2002; Manchester et al., 2004; Tóth et al., 2005). These tasks are supporting the space weather forecasting effort. The origin and evolution of a few prominent CME events has been simulated successfully on the basis of 3-D MHD simulations (Tóth et al., 2007; Lugaz et al., 2007; Manchester et al., 2008). By contrast, this work does not intend to re-enact an actually observed CME scenario but focuses rather on the fundamental processes of interaction between magnetic clouds and a structured ambient solar wind in an MHD description. Magnetic handedness forms a central concern of this paper. It is defined by the sense of rotation of force-free magnetic field lines around the MC-axis. In this way, the role of the initial magnetic handedness on the MC's evolution is emphasized. Results may be compared to similar studies performed previously by Vandas et al. $(1995,1996)$ or Schmidt and Cargill (2003), who used different numerical solvers, different grid resolutions and different models for the ambient solar wind.

In Sect. 2.1, the MHD system of equations and certain aspects concerning the applied approximate Riemann solver are discussed. Section 3 outlines the preparation of a background solar wind and introduces a simple model for the initial setup of a magnetic cloud cross section, which will be launched near the inner boundary. In Sects. 4 and 5, the results obtained for magnetic clouds propagating through the inner heliosphere for two different geometrical configurations are presented: first, for a 2-D cloud's cross section co-planar to the equatorial plane and second, for a cloud's cross section lying in a meridional plane symmetric to a heliospheric current sheet. Section 6 summarizes and discusses the results.

\section{The system of ideal MHD governing equations and its numerical solution method}

\subsection{Ideal MHD governing equations}

The transport of macroscopic quantities in a plasma is described mathematically by the set of magnetohydrodynamic (MHD) governing equations. This set of equations can be derived from kinetic theory by computing the appropriate moments of the Boltzmann equation and by subsequent summation over all particle species (electrons and protons only). The resulting single-fluid transport equations for mass, momentum and energy are augmented by Ohm's law (transport of current density) and by the four Maxwell equations. Only the electromagnetic Lorentz-force and gravitation are taken into account as external forces. Several simplifying assumptions such as quasi-neutrality, neglect of the heat flux and viscosity (ideal gas), or assuming an ideally conducting fluid yield the transport equations of compressible ideal MHD (Bittencourt, 2004). This system comprises the conservation equations for mass density, momentum density, magnetic induction and energy density. The source term is composed of a gravitational part and an additional term proportional to $\nabla \cdot \boldsymbol{B}$. The latter is proposed by Powell (1994) for an 
exclusive treatment of numerically generated $\nabla \cdot \boldsymbol{B} \neq 0$. The system of ideal compressible MHD in conservation law form reads as follows:

$$
\begin{aligned}
\frac{\partial}{\partial t}\left(\begin{array}{c}
\rho \\
\rho \boldsymbol{u} \\
\boldsymbol{B} \\
E
\end{array}\right)+\nabla \cdot\left(\begin{array}{c}
\rho \boldsymbol{u} \\
\rho \boldsymbol{u} \boldsymbol{u}+\left(p+\frac{B^{2}}{2 \mu_{0}}\right) \mathbf{I}-\frac{\boldsymbol{B} \boldsymbol{B}}{\mu_{0}} \\
\boldsymbol{u} \boldsymbol{B}-\boldsymbol{B} \boldsymbol{u} \\
\left(E+p+\frac{B^{2}}{2 \mu_{0}}\right) \boldsymbol{u}-\frac{\boldsymbol{B}(\boldsymbol{u} \cdot \boldsymbol{B})}{\mu_{0}}
\end{array}\right)= \\
=\rho\left(\begin{array}{c}
0 \\
\boldsymbol{g} \\
\mathbf{0} \\
\boldsymbol{u} \cdot \boldsymbol{g}
\end{array}\right)-\left(\begin{array}{c}
0 \\
\frac{1}{\mu_{0}} \boldsymbol{B} \\
\boldsymbol{u} \\
\frac{1}{\mu_{0}} \boldsymbol{u} \cdot \boldsymbol{B}
\end{array}\right) \nabla \cdot \boldsymbol{B},
\end{aligned}
$$

with mass density $\rho$, bulk velocity $\boldsymbol{u}$, magnetic induction $\boldsymbol{B}$, unity tensor $\mathbf{I}$, permeability of vacuum $\mu_{0}$, gravitational acceleration $\boldsymbol{g}$, and the total energy density $E$ given as

$E=\frac{\rho u^{2}}{2}+\frac{B^{2}}{2 \mu_{0}}+\frac{p}{\gamma-1}$.

So, $E$ is a sum of the kinetic, the magnetic, and the thermal energy densities, with $p$ as the thermal pressure and $\gamma$ as the polytropic index. The polytropic index is assumed to be constant across the whole computation domain and it is set to $\gamma=1.48$. A $\gamma<5 / 3(\approx 1.67)$ mimics a moderately heated solar wind plasma (Totten et al., 1995).

\subsection{Numerical solution method}

The set of compressible ideal MHD governing equations presented in Eq. (1) is composed of eight nonlinear first-order partial differential equations (PDEs) of the hyperbolic type (Toro, 1999; LeVeque, 2002). These equations have to be solved for the eight unknown parameters $\rho, \boldsymbol{u}, \boldsymbol{B}$ and $E$ (or $p$, respectively). Therefore, quantities are normalized, i.e., they are divided by a typical value for that quantity arising from the physical problem under investigation. This avoids numerical difficulties arising from extremely large or small values.

We work in the standard spherical polar coordinate system $(\mathrm{r}, \theta, \phi)$ where $\theta$ is the angular distance from the pole and $\phi$ is the azimuthal angle. The computational domain is divided into a 2-D spherical polar grid of cells ranging in radial direction from the outer regions of the solar corona at $r=0.05 \mathrm{AU}$ up to a distance beyond Earth (1.75 AU). The second dimension either creates an equatorial or a meridional plane depending on the investigated scenario of MC propagation. So, actually the propagation of a 2-D cross section of the magnetic cloud through the inner heliosphere is the subject to MHD simulations. Nevertheless, all three vector components are taken into account yielding a so-called 2.5dimensional approach. The opening angle of the second dimension is set to $100^{\circ}$ in order to account for the substantial expansion of the MC during propagation. The grid resolution in the radial direction is $\Delta \mathrm{r}=0.0025 \mathrm{AU}$, and in azimuthal or polar direction it is $\Delta \phi=\Delta \theta=0.5^{\circ}$. This constructs a polar grid consisting of $681 \times 3 \times 200$ or $681 \times 200 \times 3$ cells including also the boundary cells.

The system of governing equations is discretized in its integral form, which is achieved by integrating the differential form as presented in Eq. (1) over the control volume $\Delta t \Delta V$. This yields a so-called "finite volume" approximation. In comparison to the differential form, the integral form better reflects the physical conservation principles, and it requires less smoothness of the solution. Thus, the integral form should be preferred if solutions are expected to become discontinuous, e.g., at MHD shocks. Parameters are defined as being constant inside a cell, representing a cell average. Numerical fluxes are defined at the cell interfaces. Following an approach from Godunov (1959), these interface fluxes are computed on the basis of solutions to a local Riemann problem. Furthermore, instead of solving the original nonlinear system of PDEs, a Roe-type approximate Riemann solver is applied to the linearized version of the system (Roe, 1981; Brio and Wu, 1988; Zachary and Colella, 1992). Eigenvalues and eigenvectors for the linearized system have been calculated in terms of the primitive variables $(\rho, \boldsymbol{u}, \boldsymbol{B}, p)$ by Powell (1994, 1999). They describe eight possible waves: one entropy wave, two Alfvén waves, two slow and two fast magneto-acoustic waves, and one divergence wave. The divergence wave results from the additional source term proportional to $\nabla \cdot \boldsymbol{B}$. It ensures that any numerically generated $\nabla \cdot \boldsymbol{B}$ is convected away with the fluid. The eigenvectors for the two slow magneto-acoustic waves are transformed into an alternative form in order to avoid difficulties arising from limited floating point accuracy if $B_{x} \approx 0$, i.e., if the magnetic field component normal to the local interface vanishes.

\section{Initial conditions for the ambient solar wind and the magnetic cloud}

\subsection{The steady-state solar wind}

The inner boundary of the grid is placed at the outer regions of the solar corona, i.e., at $r=0.05 \mathrm{AU}\left(10.75 R_{\text {sun }}\right)$. This enables the assumption of an initial circular cross section for the magnetic flux tube (as will be explained in the next Sect. 3.2) and avoids taking into account effects arising from hot plasmas and resistive MHD of the inner corona. Possible viscous effects are entirely due to numerical diffusion resulting from limited grid resolution in combination with the first order accurate Riemann solver. A background solar wind is generated by setting values for the solar wind plasma at the inner boundary which then relax into a vacuum grid. Most of the parameters are kept constant at the inner boundary at each time step, and they are adjusted properly in order to create plausible solar wind conditions at $1 \mathrm{AU}$ (see, e.g., Lopez, 1987; Schwenn and Marsch, 1990; Gazis et al., 1994; Richardson et al., 1995; Paularena et al., 1998; Richardson 
Table 1. Values set for the solar wind at the inner boundary at $0.05 \mathrm{AU}$ (1st row), values gained from the simulation at $1 \mathrm{AU}$ ( $2 \mathrm{nd}$ row), and typical quiet solar wind conditions (3rd row).

\begin{tabular}{rccccccc}
\hline$(\gamma=1.48)$ & $n\left[\mathrm{~cm}^{-3}\right]$ & $u\left[\mathrm{~km} \mathrm{~s}^{-1}\right]$ & $B[\mathrm{nT}]$ & $p[\mathrm{nPa}]$ & $M_{\mathrm{A}}$ & $M_{\mathrm{S}}$ & $\beta$ \\
\hline set at 0.05 AU: & 6500 & $400\left(u_{r}\right)$ & $1160\left(B_{\mathrm{r}}\right)$ & 120.5 & 1.27 & 3.12 & 0.22 \\
computed for 1.0 AU: & 15.6 & 437 & 4.24 & 0.0204 & 18.6 & 12.9 & 2.8 \\
typical for 1.0 AU: & 10.0 & 450 & 6.00 & 0.020 & 10.0 & 10.0 & 1.3 \\
\hline
\end{tabular}

and Smith, 2003; Wang and Richardson, 2004; or Liu et al., 2006). The polytropic index is set to $\gamma=1.48$ for the entire computation domain. This mimics a moderately heated solar wind (Totten et al., 1995). The selection of inner boundary values and the results from the MHD simulation gained at $1 \mathrm{AU}$ are listed in Table 1. These 2-dimensional computations are performed in an equatorial plane, so, no heliospheric current sheet has to be taken into account. A direct comparison with typical values for the solar wind of the quiet Sun (listed in the third row of Table 1) shows a relatively good match between real conditions and results from numerical simulations. The Alfvén and sonic Mach numbers, $M_{A}$ and $M_{S}$, from the simulation at $1 \mathrm{AU}$ are a little bit too high, which is mainly caused by an overestimated plasma density $n$ at the inner boundary $\left(6500 \mathrm{~cm}^{-3}\right.$ instead of $4000 \mathrm{~cm}^{-3} \mathrm{ac}-$ cording to the theoretical $1 / r^{2}$-decay). This is due to the fact that $M_{\mathrm{A}}$ at the inner boundary has to be $>1$ (super-Alfvénic flow) for a smooth dependence of $B_{\phi}$ with radial distance, i.e., to get a smooth Parker spiral IMF. The Parker spiral in the IMF is realized by setting $B_{\phi}$ to its nominal value at the inner boundary (Parker, 1958, 1963). The radial components of velocity and the magnetic field, $u_{\mathrm{r}}$ and $B_{\mathrm{r}}$, are set to the values listed in Table $1\left(400 \mathrm{~km} \mathrm{~s}^{-1}\right.$ and $1160 \mathrm{nT}$, respectively). A small azimuthal velocity component is allowed to adjust itself by setting $u_{\phi}$ as a free parameter (zero-derivative at the inner boundary). The meridional components of $\boldsymbol{u}$ and $\boldsymbol{B}$, i.e., $u_{\theta}$ and $B_{\theta}$, are kept zero at the inner boundary.

If conditions in a meridional plane are simulated, the following upgrades have to be introduced. First, the absolute values of solar wind parameters become a function of the polar distance angle. The velocity and the magnetic field strength are higher near the poles than near the equator. With regard to the wind speed, this is particularly true during a solar minimum phase where one typically has a bimodal wind with slow wind near the ecliptic and fast wind at high latitudes. Furthermore, the density decreases towards the poles and the thermal pressure stays nearly constant. The meridional dependence of parameters $n, u$, and $p$ at the inner boundary have been adjusted according to Ulysses observations as outlined in Roussev et al. (2003). The latitudinal dependence of the magnetic field strength is set according to a dipole-like model. Furthermore, $\boldsymbol{B}_{\mathrm{IMF}}$ is oriented in opposite directions across the equator thereby forming a heliospheric current sheet (HCS) at the equatorial plane. For simplicity, it is assumed that there is no tilt between the Sun's magnetic and rotational axes. The HCS is characterized by an enhanced thermal pressure and by a decrease in magnetic field strength and velocity. Observations have shown that the total pressure ( $p_{\text {tot }}=p_{\text {therm }}+p_{\text {mag }}$ ) usually stays constant across the HCS (Winterhalter et al., 1994), so that the HCS may be interpreted as a tangential discontinuity of exceptional thickness. The development of a HCS is made possible by defining a narrow $\theta$-range around the equator inside which boundary parameters are not kept on their initial values, but they are adjusted partially by the simulation (zeroderivative at boundary). The HCS remains stable only if the polar components $u_{\theta}$ and $B_{\theta}$ are kept zero, and if $B_{\mathrm{r}}$ is adjusted inside the HCS so that $\nabla \cdot \boldsymbol{B}=0$ is fulfilled. A zoom into the meridional grid near the inner boundary is presented in Fig. 1, when an MHD steady-state for the solar wind has been achieved after several thousand time steps.

\subsection{The initial magnetic cloud}

After having completed the simulation of the solar wind flow from the inner boundary at $0.05 \mathrm{AU}$ to the outer boundary at $1.75 \mathrm{AU}$, each cell of the grid from the last time step is filled with proper plasma and magnetic field parameters representing an average solar wind during a solar minimum phase. As a next step, a magnetic cloud is placed near the inner boundary onto the solar wind grid. Since computations are performed only in two dimensions, the magnetic cloud is actually modeled as a two-dimensional cross section of the threedimensional flux rope, either in an equatorial or in a meridional plane. Near the Sun, this cross section is taken to be circular. In the meridional view, it is placed symmetrically with regard to the heliospheric current sheet as if the flux rope emerges exactly above the helmet streamer belt (Pneumann and Kopp, 1971; Low, 1996). The grid cells inside this cross section have to be filled with plausible values for the plasma and the magnetic field in order to mimic a magnetic cloud near the Sun. The strategy for setting up the initial status for a magnetic cloud can be outlined as follows (see also Fig. 1):

- The magnetic cloud is modeled as a circular cross section with an initial radius of $\sim 8 R_{\text {sun. }}$. The center of the cross section is placed near the inner boundary at $r=0.1 \mathrm{AU}$. 

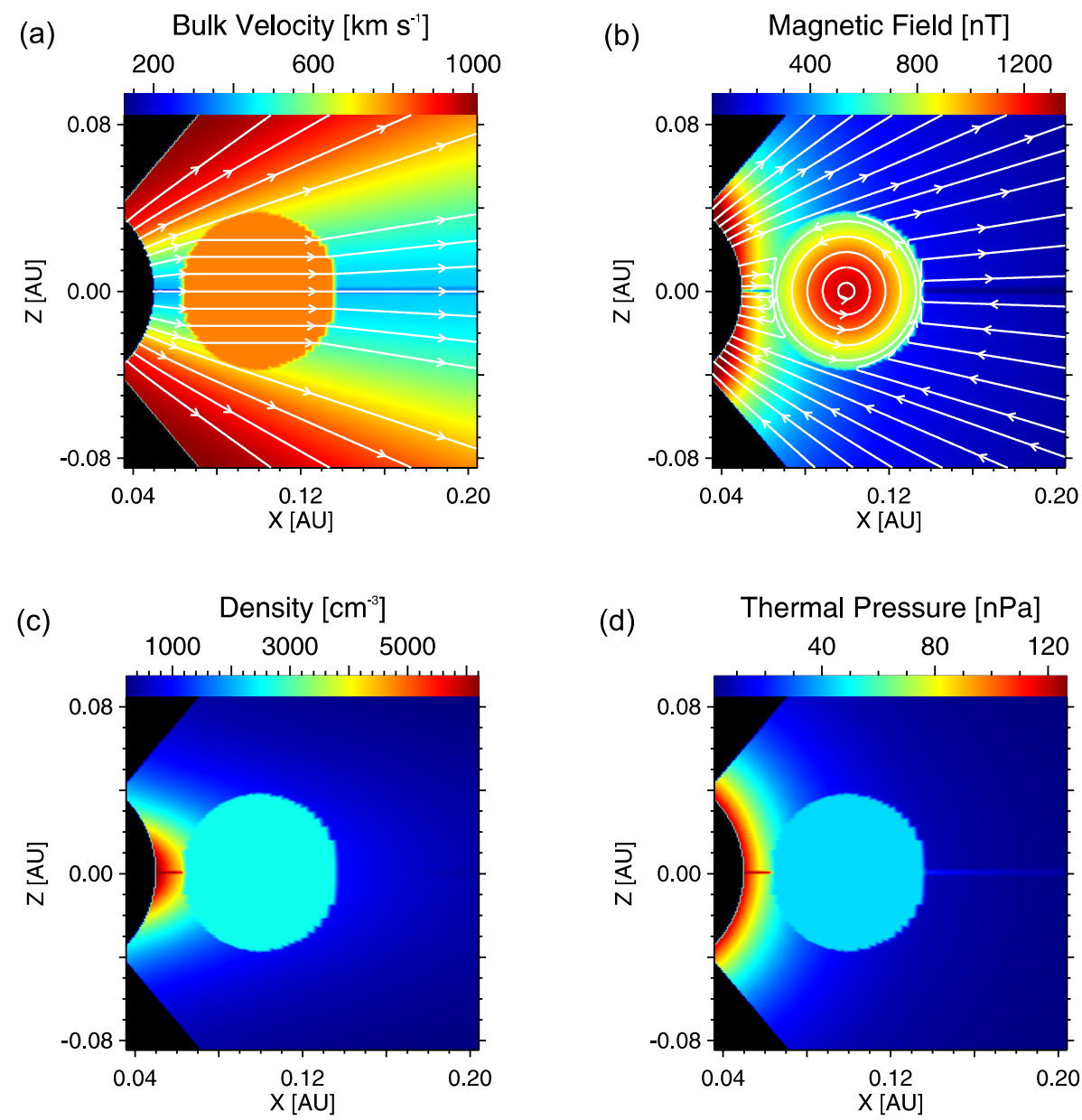

Fig. 1. Distributions of plasma speed (a), magnetic field (b), number density (c) and thermal pressure (d) for the initial state of the background solar wind and for the initial circular magnetic cloud cross section. Distributions are shown in a meridional plane near the inner boundary of the grid. Streamlines for velocity and field lines for the magnetic field are over-plotted as a projection onto the plane of viewing.

- For simplicity, $\rho$ and $p$ inside the MC cross section are set constant. It was decided to use initial values for $\rho$ and $p$ which approximately correspond to mean values derived from the upstream and downstream ambient solar wind. So, $n_{\mathrm{mc}} \approx 2600 \mathrm{~cm}^{-3}$ and $p_{\mathrm{mc}} \approx 45 \mathrm{nPa}$.

- The plasma speed inside the cross section is constant as well, so that there is actually one velocity vector which represents the velocity of the whole MC cross sectional area. This avoids possible expansion of the MC triggered by initially diverging velocities. The velocity vectors in each cell of the cross section point into positive $\mathrm{x}$-direction, and the absolute velocity is set to $u_{\mathrm{mc}}=800 \mathrm{~km} \mathrm{~s}^{-1}$.

- The initial magnetic field inside the circular cross section is set up as a constant- $\alpha$ force-free magnetic field with cylindric geometry (Lundquist, 1950; Lepping et al., 1990). Many observations performed by various spacecraft have confirmed the assumption of a forcefree field, at least for a local view, if the large-scale curvature of the cloud can be neglected. Force-free means that currents $\boldsymbol{j}$ point into the direction of $\boldsymbol{B}$, i.e., $\boldsymbol{j} \sim \nabla \times \boldsymbol{B}=\alpha \boldsymbol{B}$. The quantity $\alpha$ is a scaling parameter for the size of the force-free region. The magnetic field components for a force-free field in a cylindric coordinate system, with its center coinciding with the center of the circular cross section, are defined as:

$B_{\mathrm{r}}=0$,

$B_{\phi}=B_{0} H J_{1}(\alpha r)$,

$B_{\mathrm{Z}}=B_{0} J_{0}(\alpha r)$.

Quantity $B_{0}$ is the magnetic field strength on the axis of the cylindric magnetic cloud. It is set to $B_{0} \approx 1280 \mathrm{nT}$ (see, e.g., radial fit in Leitner et al., 2007). Quantities $J_{0}$ and $J_{1}$ are the zero and first order J-Bessel functions 

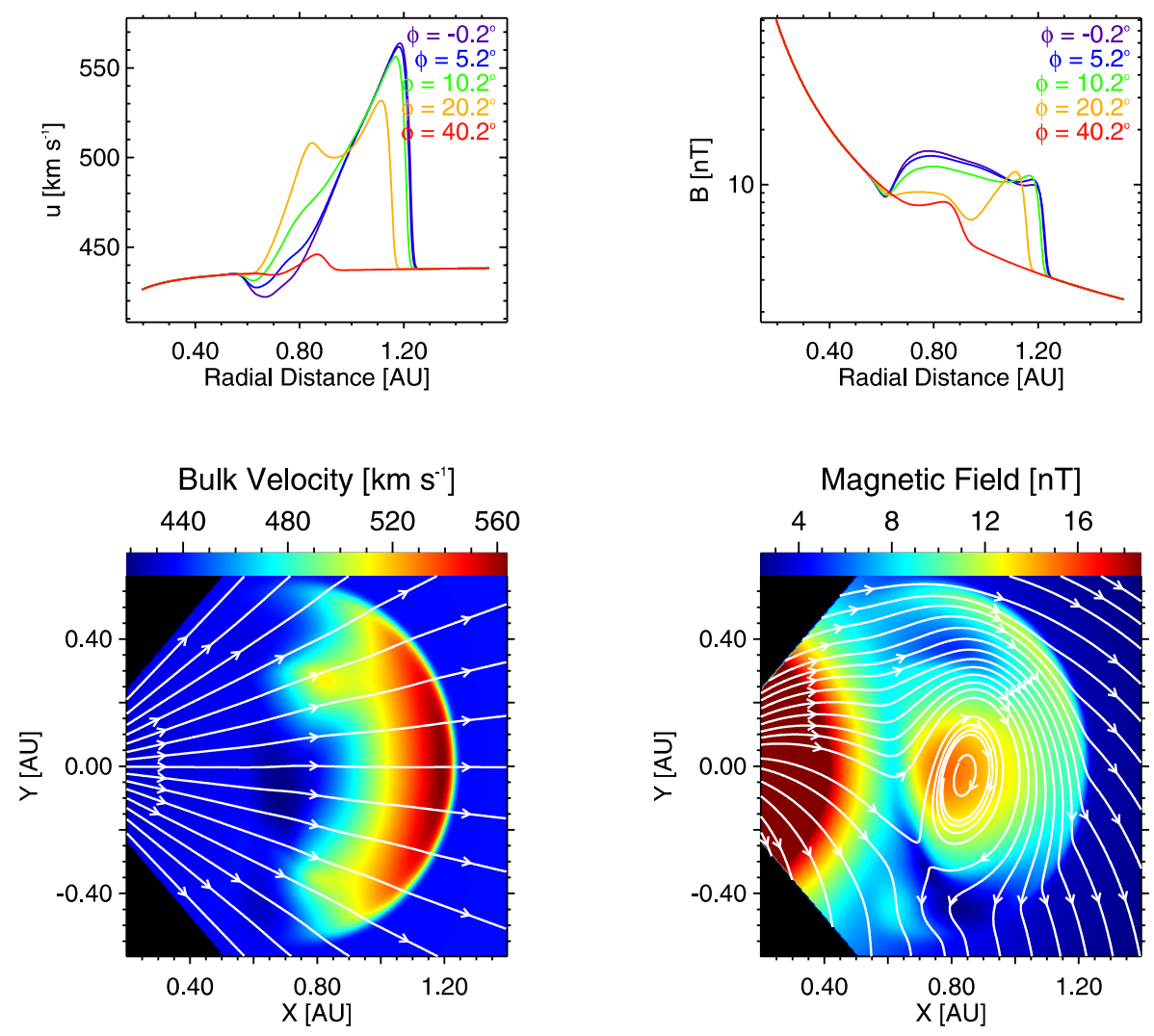

Fig. 2. Velocity distribution (bottom left) and magnetic field distribution (bottom right) for the $H_{\mathrm{e}}^{-}$magnetic cloud at the distance of Earth (MC-center at $\sim 0.84 \mathrm{AU}$ ). Corresponding profiles at constant azimuthal angels are shown in the top row. Velocity streamlines and magnetic field lines are over-plotted in white color, respectively.

which define the behavior of $B_{\phi}$ and $B_{\mathrm{Z}}$ as a function of distance $r$ to the cloud's center. The boundary of the circular cloud cross section is placed where $B_{\mathrm{Z}}$ and $J_{0}$ become zero, respectively. So, $\alpha$ has to be set to $\alpha=2.4047 / r_{\mathrm{mc}}$, with $r_{\mathrm{mc}}$ as the cloud's radius. Finally, the parameter $H$ defines the handedness of the magnetic field. $H=+1$ generates a positive $B_{\phi}$, i.e., magnetic field lines rotate counter-clockwise around the center if viewed from a positive $\mathrm{z}$-location towards the center. On the contrary, $H=-1$ generates a clockwise rotating magnetic field if viewed from a positive z-location towards the center. All possible combinations of positive or negative $B_{\phi}$ and $B_{\mathrm{Z}}$ have been observed (Bothmer and Schwenn, 1998).

The initial setup for the parameters $n, \boldsymbol{u}, \boldsymbol{B}$ and $p$ in a meridional plane is presented in Fig. 1. It shows a zoom into the computation domain near the inner boundary ranging from $r=0.05-0.20 \mathrm{AU}$. The initial circular cross section of the $\mathrm{MC}$ is placed into the bi-modal background solar wind symmetric about the heliospheric current sheet (at $z=0$ ). In the Northern Hemisphere, IMF field lines are pointing away from the Sun, and in the Southern Hemisphere they are pointing towards the Sun. At the current sheet, the velocity and the magnetic field strength are decreasing whereas the plasma density and thermal pressure are increasing. Inside the MC, the initial values for $n, p$ and $u$ are set constant. The magnetic field of the cloud corresponds to a force-free field with cylindric geometry. We are illustrating the case when the handedness is +1 , and $B_{\phi}$ along the cloud's axis is pointing away from the observer.

\section{Evolution of the MC cross section in the equatorial plane}

This section presents the results, which are obtained for the propagation of a magnetic cloud whose axis is oriented perpendicular to the equatorial plane. The evolution of the MC cross section in the equatorial plane is simulated for two cases of opposite handedness of the initial force-free magnetic field, i.e., $H=-1$ and $H=+1$ (see Eq. 4). For convenience, the expressions " $H_{\mathrm{e}}^{--}$" and " $H_{\mathrm{e}}^{+}$" are introduced to distinguish between those two cases of magnetic configurations in the equatorial plane. All other initial parameters of the two MCs, concerning the magnetic field strength and the state of the plasma, are the same. 

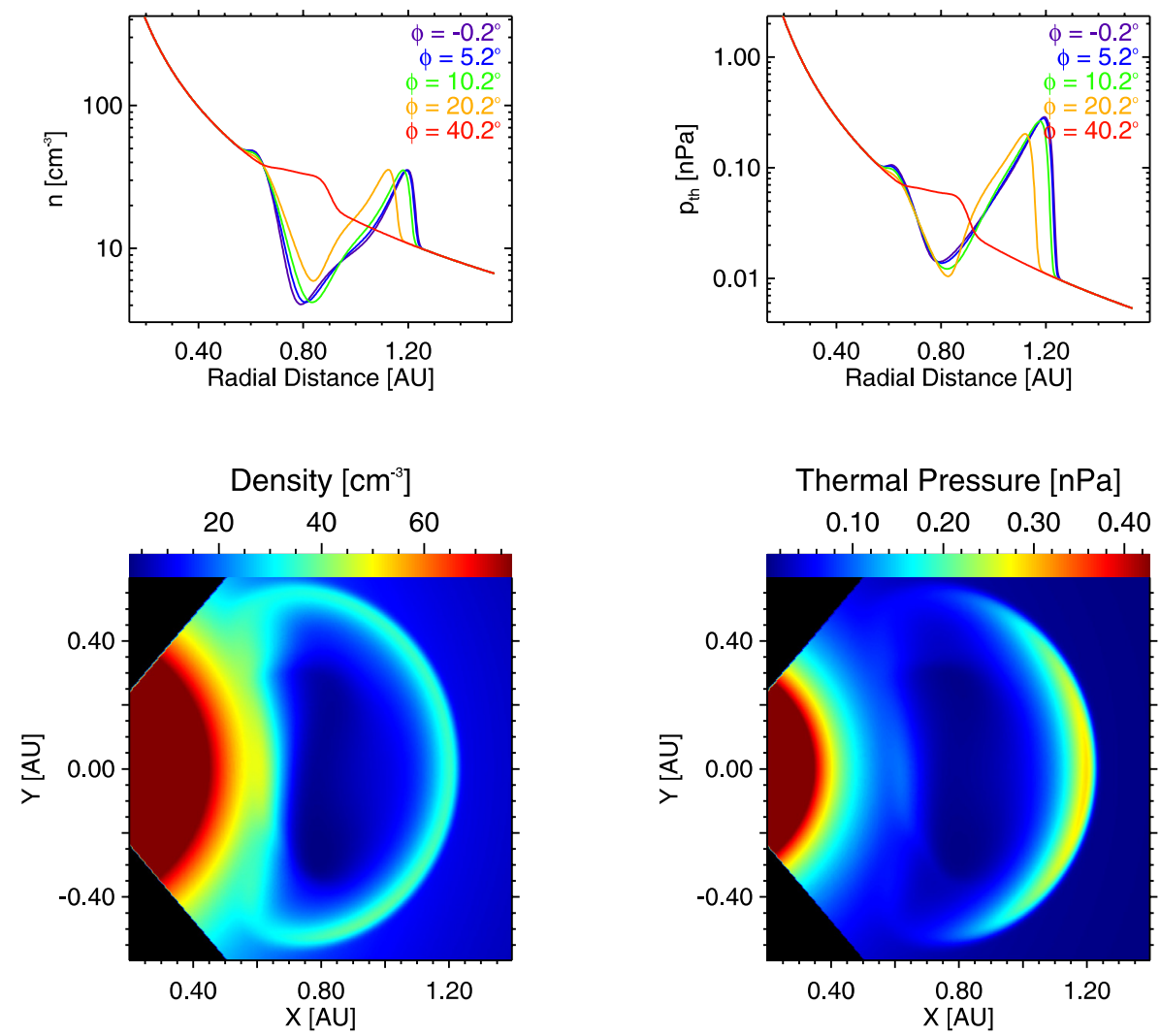

Fig. 3. Density (left) and thermal pressure (right) for the $H_{\mathrm{e}}^{-}$magnetic cloud near Earth.

Results obtained after $\sim 63 \mathrm{~h}$ of MC propagation are presented for the distributions of velocity and the magnetic field in Fig. 2 and in Fig. 4, respectively. Figure 3 and Fig. 5 show the distributions of number density and thermal pressure. Radial profiles at selected azimuthal angles are also included in the top rows of these figures. Amplitudes are color coded, and a projection of field lines onto the 2-D grid is over-plotted for the vector quantities $\boldsymbol{u}$ and $\boldsymbol{B}$. The centers of $H_{\mathrm{e}}^{-}$and $H_{\mathrm{e}}^{+}$can be found around $0.85 \mathrm{AU}$. The significant similarities and differences between $H_{\mathrm{e}}^{-}$and $H_{\mathrm{e}}^{+}$seen in the MHD simulations can be summarized as follows.

Both magnetic clouds are subject to substantial expansion, which is initially caused by an excess of magnetic pressure. Later on, expansion is driven by a stronger decay of total pressure in the ambient solar wind than inside the magnetic cloud. Placed into an ambient flow with constant speed, the MCs are decelerated in radial direction due to the hydrodynamic drag. At the same time, they tend to maintain their angular extent thus becoming elongated in the azimuthal direction (Newkirk et al., 1981). Thereby, the cross sectional shape is changed from circular to approximately elliptical (see contours of magnetic field lines drawn in Fig. 2 and in Fig. 4).
Due to expansion, the interiors of both MCs have been depleted from plasma leading to a lower mass density and thermal pressure as compared to the ambient solar wind (see Fig. 3 and Fig. 5). By contrast, the magnetic field strength still exceeds the Parker spiral IMF strength yielding a low- $\beta$ plasma inside the MC cross sections.

The supersonic flow generates a fast mode shock and a sheath ahead of both MCs (Erkaev et al., 1995; Siscoe and Odstrcil, 2008). For a fast mode shock, the shock speed, measured relative to the speed of the ambient solar wind, has to exceed the local fast magneto-acoustic wave speed. For example, at the shock front of $H_{\mathrm{e}}^{-}$near $x=1.2 \mathrm{AU}$, $y=0.0 \mathrm{AU}$, the relative shock speed peaks at $125 \mathrm{~km} \mathrm{~s}^{-1}$ $\left(=563 \mathrm{~km} \mathrm{~s}^{-1}-438 \mathrm{~km} \mathrm{~s}^{-1}\right)$ which clearly exceeds the local fast magneto-acoustic wave speed of $\sim 85 \mathrm{~km} \mathrm{~s}^{-1}$. At the shock front, the solar wind plasma becomes compressed and heated. This is clearly visible according to the enhancements of density and thermal pressure shown in Fig. 3 and in Fig. 5. The heating in collisionless shocks is due to a dissipative process, e.g., wave-particle interactions. Dissipation leads to a transfer of kinetic energy into heat and furthermore, to particle acceleration at the shock. Another characteristic feature of a fast mode shock is the fact that if one crosses the shock from downstream, magnetic field lines are always bent away from the shock normal. 

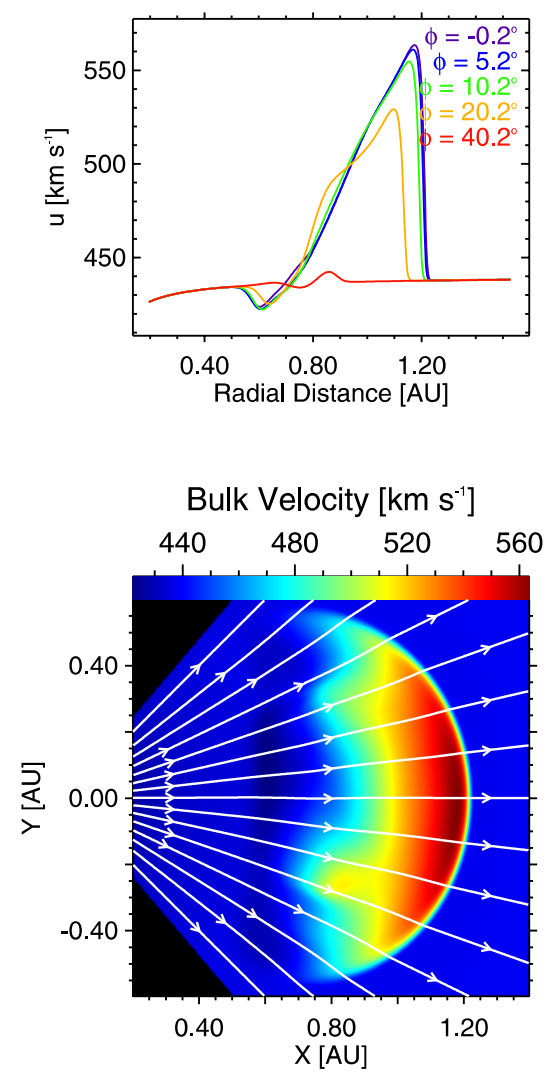
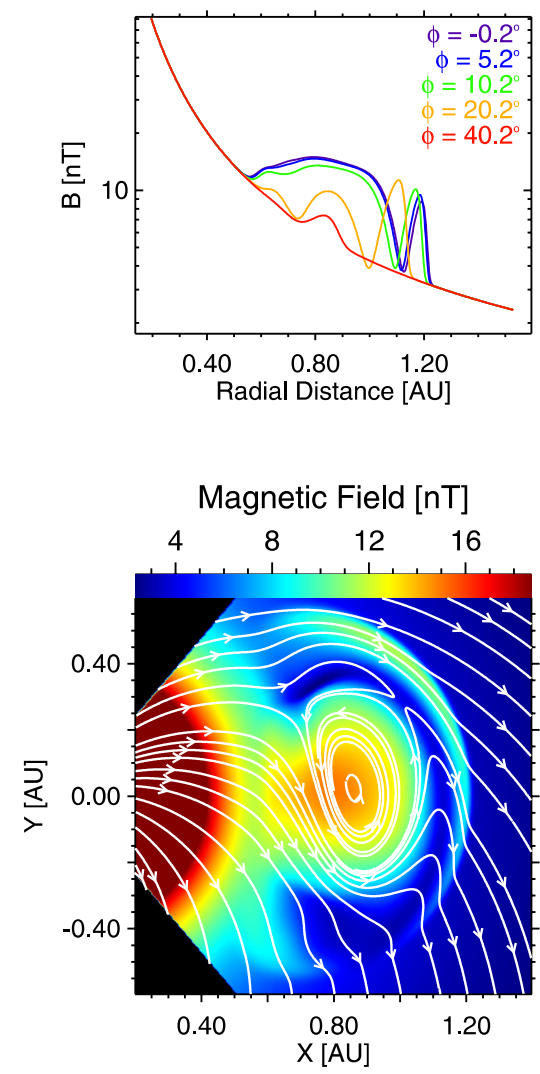

Fig. 4. Velocity (left) and magnetic field (right) for the $H_{\mathrm{e}}^{+}$magnetic cloud near Earth.

For both magnetic clouds, the shock fronts already passed Earth, so, after $63 \mathrm{~h}$ of the simulation, Earth would be placed somewhere inside the sheath. Note that at about $1 \mathrm{AU}$ distance to the Sun, the shock front spans an azimuthal angle of almost $90^{\circ}$. Furthermore, there is a rarefaction wave visible at the rear side of both MCs caused by the expansion. This leads to a shocked solar wind plasma at the backside as well (see small humps in profiles for $n$ and $p_{\text {th }}$ in Fig. 3 and Fig. 5, located at $r \sim 0.60 \mathrm{AU})$. For $H_{\mathrm{e}}^{+}$, the magnetoplasma parameters at this reverse shock show the same behavior as already reported by Gosling et al. $(1994,1998)$ for over-expanding coronal mass ejections. For $H_{\mathrm{e}}^{-}$, the magnetic field exhibits a decrease instead of an increase if crossing the reverse shock from upstream. This is due to magnetic reconnection occurring on the backside of $H_{\mathrm{e}}^{-}$, a phenomenon which will be discussed in more detail later on.

The expansion of both magnetic clouds is analyzed in two different ways. First, the MC boundaries can be considered as the outermost contours of 2-D magnetic field lines which have been projected into the equatorial plane. The evolution of these outermost contours as a function of time is shown in Fig. 6a for $H_{\mathrm{e}}^{-}$and in Fig. $6 \mathrm{~b}$ for $H_{\mathrm{e}}^{+}$. Time is encoded in rainbow colors on the contours and distances are normalized to the respective MC-centers. The simplifying assump- tion of ignoring gradients of $\boldsymbol{B}$ into the third dimension $(\theta$ dimension), together with the possibility of magnetic reconnection between the IMF and the MC, introduces substantial distortions on projected field lines, making it impossible to find a "closed" line near the MC boundary. Instead, 2-D field lines of the MC interior look as if they are spiraling inward towards the center. So, the boundary of the MC was defined as the contour of the first field line starting to spiral towards the center when approaching the MC from outside. Ellipses may be approximated to these contours yielding, after $63 \mathrm{~h}$ of propagation, numerical eccentricities of $\varepsilon=\sqrt{a^{2}-b^{2}} / a=$ 0.810 for $H_{\mathrm{e}}^{-}$(semi-major axis $a \approx 0.265 \mathrm{AU}$, semi-minor axis $b \approx 0.156 \mathrm{AU})$, and $\varepsilon=0.770$ for $H_{\mathrm{e}}^{+}(a \approx 0.281 \mathrm{AU}$, $b \approx 0.180 \mathrm{AU})$. The eccentricity $\varepsilon$ is increasing with increasing distance from the Sun, thus, the elliptical shape becomes more and more pronounced.

Furthermore, expansion has been analyzed on a hydrodynamic basis by trying to get insight into expansion velocities relative to the MC-center. Plots for these expansion velocities of $H_{\mathrm{e}}^{-}$and $H_{\mathrm{e}}^{+}$are presented in Fig. 6c and 6d. Both images depict the various paths of 180 uncharged test particles during the simulation. These test particles are dropped into the initial grid along a ring (fluid line) closely around the initial MC-center. For each time step, particle positions 

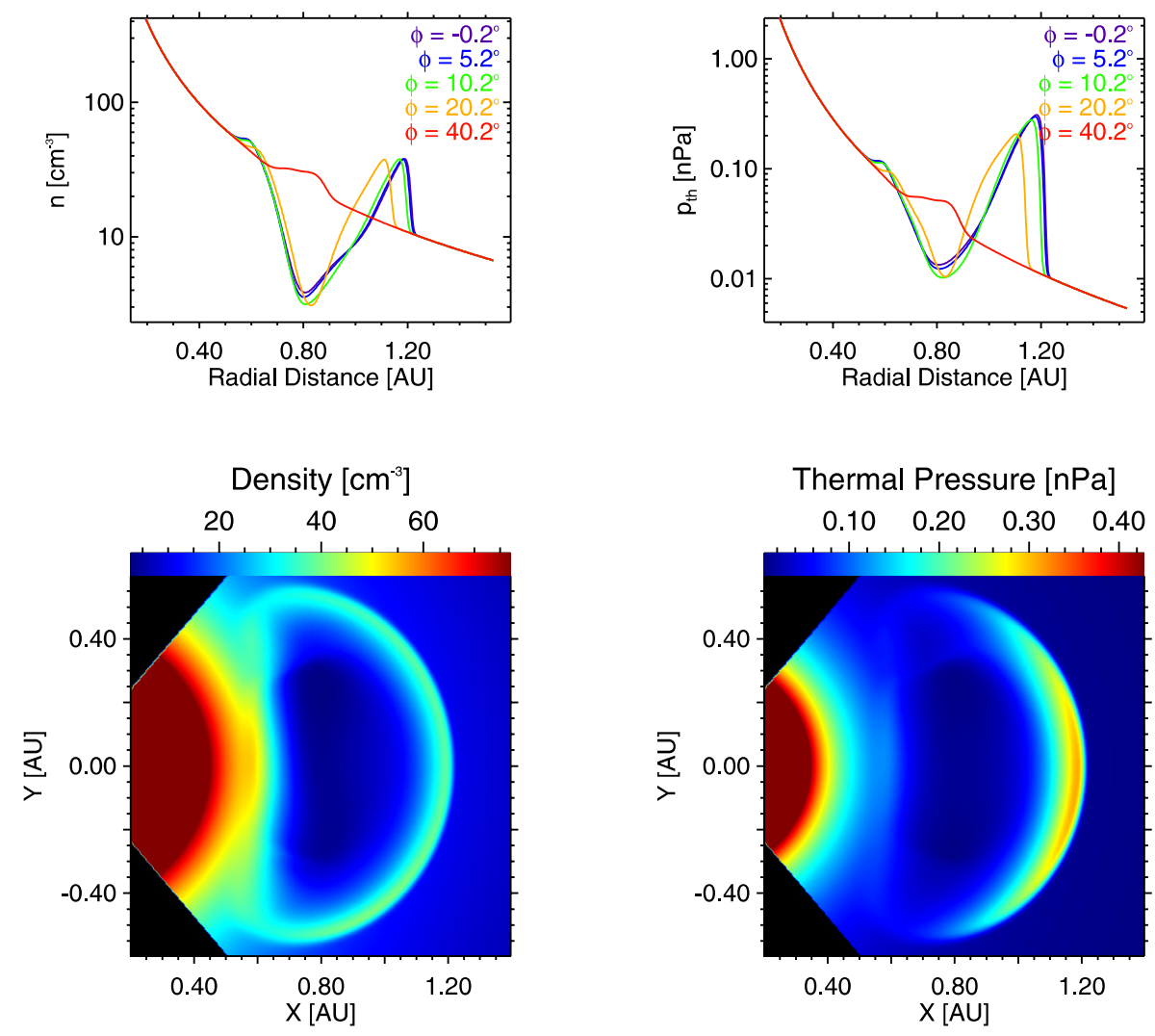

Fig. 5. Density (left) and thermal pressure (right) for the $H_{\mathrm{e}}^{+}$magnetic cloud near Earth.

are given relative to the position of the MC-center. The velocity of test particles, i.e., the hydrodynamic expansion velocities at different locations inside the MC, are color coded along the paths. It can be seen that soon after initiation of the simulation, there is a rapid expansion into azimuthal direction (red shaded areas) with a peak at $\sim 180 \mathrm{~km} \mathrm{~s}^{-1}$ for both equatorial scenarios. Even for later times at $t>20 \mathrm{~h}$, the expansion velocities in the azimuthal direction stay close to $\sim 100 \mathrm{~km} \mathrm{~s}^{-1}$ (green-yellow areas) whereas in radial direction, the expansion velocities drop below $\sim 50 \mathrm{~km} \mathrm{~s}^{-1}$ (blue shaded areas), leading to the characteristic elliptic shape. For better visualization of the expansion, fluid lines are drawn in gray color every $5 \mathrm{~h}$. Expansion speeds may also be compared to the analytical model of Owens et al. (2005), yielding $u_{\exp } \approx 78 \mathrm{~km} \mathrm{~s}^{-1}$. This analytical value is settled between the expansion speeds recovered from MHD simulation into the direction of propagation $\left(50 \mathrm{~km} \mathrm{~s}^{-1}\right)$ and perpendicular to it $\left(100 \mathrm{~km} \mathrm{~s}^{-1}\right)$.

Regarding the velocity distributions of both MCs, velocities are in general oriented in radial direction as can be seen from the streamlines over-plotted in Fig. 2 and in Fig. 4. The azimuthal and polar velocity components $u_{\phi}$ and $u_{\theta}$ are displayed in Fig. 7. Additionally, 2-D projected magnetic field lines are over-plotted in gray or white color in order to be able to estimate the locations of the MC body, the sheath and the foreshock. The components $u_{\phi}$ and $u_{\theta}$ exhibit a maximum amplitude around $35 \mathrm{~km} \mathrm{~s}^{-1}$, which is much smaller than the radial component $u_{\mathrm{r}}\left(\sim 560 \mathrm{~km} \mathrm{~s}^{-1}\right.$ at the shock). Inside the sheath ahead of both clouds, $u_{\phi}$ is directed in such a way that piled-up plasma of the slower ambient solar wind is always guided towards the flanks and furthermore around the MCbody (see Fig. 7b and 7e). The deflection velocity behind the shock front becomes even higher at greater y-distances, with peaks around $\pm 35 \mathrm{~km} \mathrm{~s}^{-1}$. Deeper inside the sheath, $u_{\phi}$ changes its sign, but magnitudes are too small to cause a serious accumulation of plasma along the stagnation line.

The grids for $u_{\theta}$, shown in Fig. 7a and 7d, reveal the presence of two antiparallel flows perpendicular to the equatorial plane inside both MCs. It should be noted that flows in $\theta$ direction may not be captured correctly by these 2.5-D simulations. Nevertheless, it can be concluded that the magnetic handedness of the cloud seems to have a fundamental influence on the direction of these polar flows: For $H_{\mathrm{e}}^{-}, u_{\theta}$ points towards south at positive y-locations, and it points towards north at negative y-locations. For $H_{\mathrm{e}}^{+}$, exactly the opposite is true, with northward flows at positive y-locations and southward flows at negative y-locations. 

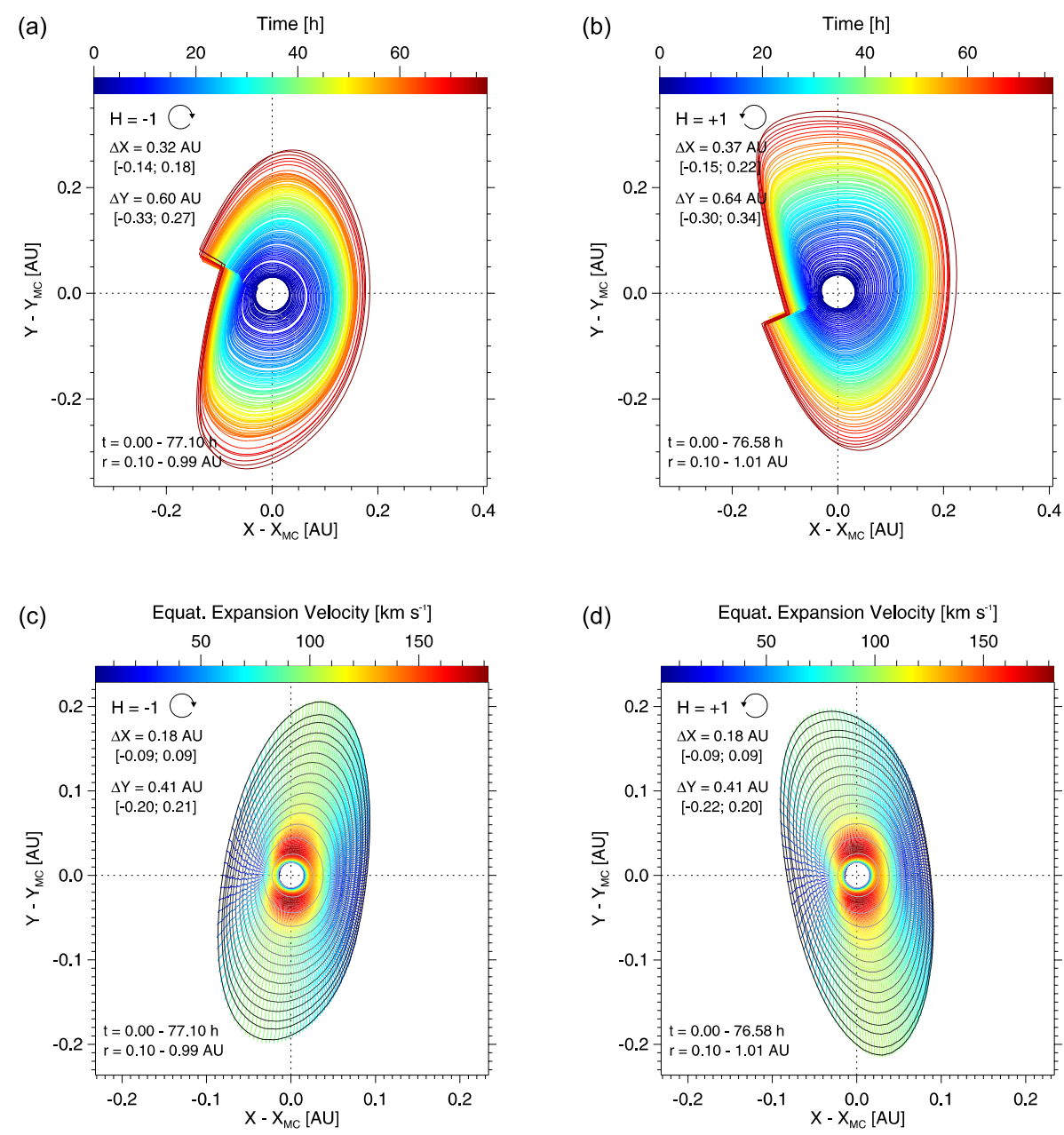

Fig. 6. Expansion of the MCs in the equatorial plane. The upper row shows the outermost "closed" magnetic field line around the MC-center at selected times of the simulation for $H_{\mathrm{e}}^{-}$(a) and $H_{\mathrm{e}}^{+}$(b). The lower row illustrates the expansion of plasma inside $H_{\mathrm{e}}^{-}$(c) and $H_{\mathrm{e}}^{+}$(d). The various paths of 180 test particles are plotted relative to the MC-center, and the expansion velocities, i.e., the velocities of test particles relative to the velocity of the MC-center, are color-coded along the paths. Gray contours (fluid lines) around the MC-center are drawn every $5 \mathrm{~h}$.

As already noted, magnetic reconnection occurs between the magnetic field of the cloud and the IMF (Erkaev et al., 2002; Dasso et al., 2007). For $H_{\mathrm{e}}^{-}$, magnetic reconnection takes place on the back side flank at negative $y$-coordinates, and for $H_{\mathrm{e}}^{+}$, magnetic reconnection takes place on the front side flank at positive y-coordinates, where orientations of IMF lines and MC field lines become antiparallel. As a consequence, there is a broad zone of decreased magnetic field strength visible inside the sheath of $\mathrm{H}_{\mathrm{e}}^{+}$. Inside this magnetic depression, the magnitude of $B_{\mathrm{IMF}}$ is falling back to a value which is usually obtained for the downstream ambient solar wind. This region is often referred to a magnetic hole if detected in in-situ observations (Farrugia et al., 2001; Lepping et al., 2009, and references therein). It should be emphasized that any magnetic reconnection seen in these MHD simulations is exclusively due to numerical diffusion effects. Thus, reconnection rates may be overestimated, especially at larger distances to the inner boundary where the resolution of the spherical grid becomes coarse. A small acceleration of MC plasma towards the reconnection site is also visible in the $u_{\phi^{-}}$ grids of Fig. 7 (amplitudes around $\pm 20 \mathrm{~km} \mathrm{~s}^{-1}$ ). As can be seen in Fig. $7 \mathrm{~b}, u_{\phi}$ of $H_{\mathrm{e}}^{-}$is mainly negative (blue) inside the MC body pointing towards the reconnection site located at negative y-coordinates. By contrast, $u_{\phi}$ of $H_{\mathrm{e}}^{+}$(Fig. 7e) is mainly positive (red) pointing towards the reconnection site located at positive y-coordinates. As will be seen later on, such an azimuthal movement of plasma inside the cloud causes a drift of the whole MC cross section towards the site of magnetic reconnection.

Another consequence of magnetic reconnection is a deceleration of plasma into radial direction near the reconnection site. This introduces a slight tilt of the cross sectional 
(a)

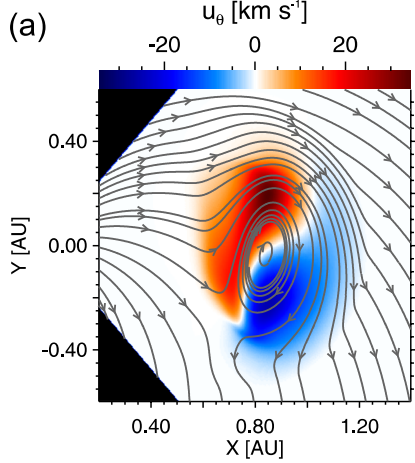

(d)

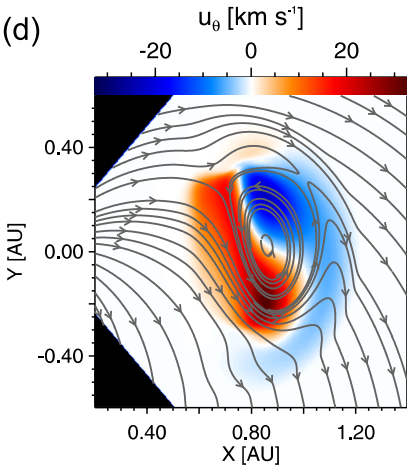

(b)

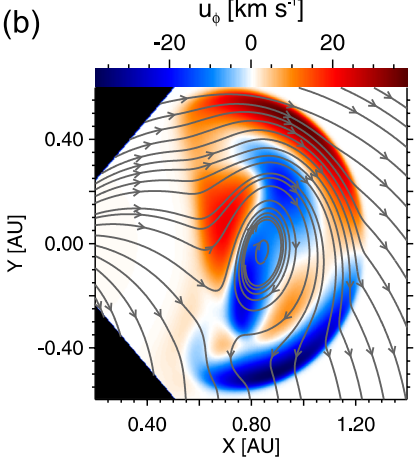

(e)

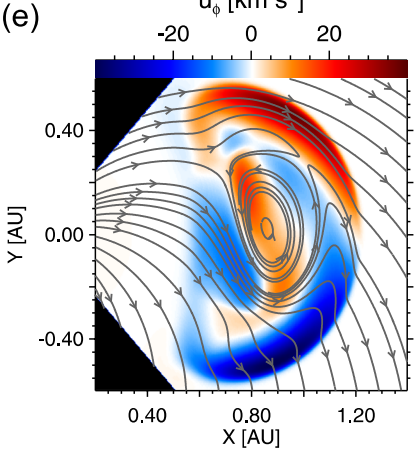

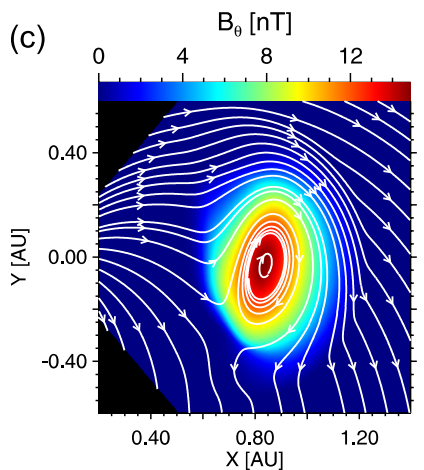
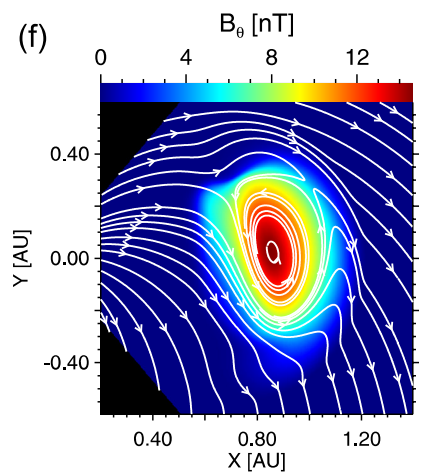

Fig. 7. Grids for the polar and azimuthal velocity components $u_{\theta}$ and $u_{\phi}$ of $H_{\mathrm{e}}^{-}(\mathbf{a}, \mathbf{b})$ and of $H_{\mathrm{e}}^{+}(\mathbf{d}, \mathbf{e})$. The polar component $B_{\theta}$ of the magnetic field is shown in (c) for $H_{\mathrm{e}}^{-}$and in (f) for $H_{\mathrm{e}}^{+}$. Projected magnetic field lines are over-plotted in gray/white color.

ellipse, i.e., a tilt of the semi-major axis away from the ydirection. In the case of $H_{\mathrm{e}}^{-}$, magnetic reconnection occurs behind the cloud. Thus, a deceleration of plasma behind the $\mathrm{MC}$ at negative $\mathrm{y}$-coordinates tilts the ellipse in clockwise direction $\left(\sim 12^{\circ}\right)$, whereas magnetic reconnection and a deceleration of plasma in front of $H_{\mathrm{e}}^{+}$at positive y-coordinates tilts the corresponding ellipse in counter-clockwise direction $\left(\sim 8^{\circ}\right)$.

Moreover, it should be noted that due to the distortions introduced by magnetic reconnection, the centers of both MCs do not coincide with the point of maximum magnetic field strength anymore (despite this having been the case for the initial grid). The point of maximum $B$ inside $H_{\mathrm{e}}^{-}$and $H_{\mathrm{e}}^{+}$ is found to be $0.06 \mathrm{AU}$ offset behind the cloud's axis (after $63 \mathrm{~h}$ of propagation). However, the $B_{\theta}$-component, which is the component oriented perpendicular to the equatorial plane, still has its maximum located exactly on the axis of both clouds (see Fig. 7c, f).

The evolution of sizes and shapes for $H_{\mathrm{e}}^{-}$and $H_{\mathrm{e}}^{+}$is illustrated in Fig. 8. Figure 8a shows the extensions of $H_{\mathrm{e}}^{-}$(blue traces) and $H_{\mathrm{e}}^{+}$(red traces) into x-direction, i.e., into the direction of propagation, along $y=Y_{\mathrm{MC}-\text { center. }}$. It demonstrates again the influence of magnetic reconnection on the evolution of the clouds' boundaries. The extension towards the backside is approximately the same for $H_{\mathrm{e}}^{-}$and $H_{\mathrm{e}}^{+}$, apart from the fact that this extension is hard to estimate because magnetic field lines have a smooth transition to the IMF on the backside. On the front side, $H_{\mathrm{e}}^{+}$exhibits a larger extension than $H_{\mathrm{e}}^{-}$because magnetoplasma of $H_{\mathrm{e}}^{+}$is accelerated towards the site of magnetic reconnection which is located in the downstream sheath. At the same time, the shocked solar wind is accelerated from the shock front upstream into the sheath, thereby reducing the shock stand-off distance. Thus, it can be concluded that at $1 \mathrm{AU}$ distance from the Sun, $\mathrm{H}_{\mathrm{e}}^{+}$ has a larger extension into the direction of propagation than $H_{\mathrm{e}}^{-}$, but the latter would be detected earlier at Earth due to its greater shock stand-off distance ( $0.28 \mathrm{AU}$ vs. $0.20 \mathrm{AU})$. Figure $8 \mathrm{~b}$ indicates the shift of the clouds' $y$-boundaries towards positive y-coordinates for $H_{\mathrm{e}}^{+}$and towards negative y-coordinates for $H_{\mathrm{e}}^{-}$, which is caused by an acceleration of plasma perpendicular to the magnetic field towards the reconnection site. In turn, plasma is also expelled from the reconnection site into the direction of the original magnetic field orientation.

The total extension into x-direction for both MCs is presented in Fig. 8c. It is arranged around $0.3 \mathrm{AU}$ at $1 \mathrm{AU}$ distance from the Sun, which is in good agreement with common values found in the literature (Lepping et al., 1990; Hu and Sonnerup, 2002). The total extension into y-direction at $1 \mathrm{AU}$, i.e., the extension perpendicular to the direction of 


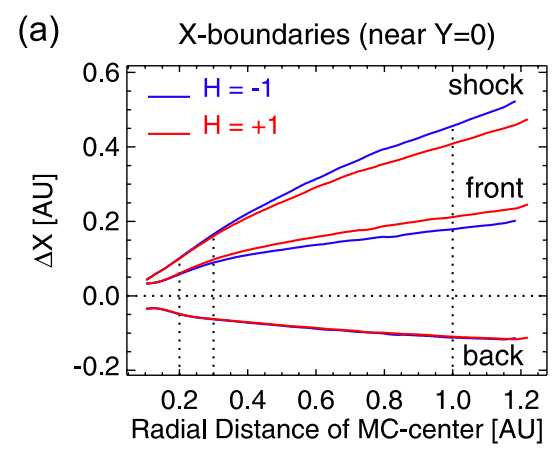

(c)

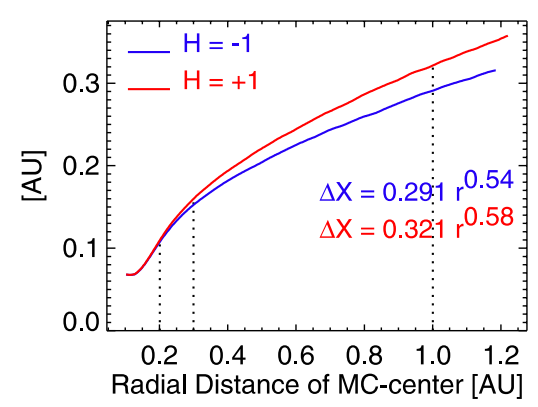

(b)

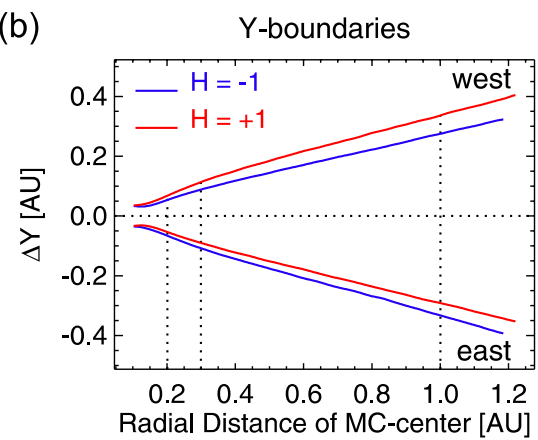

(d)

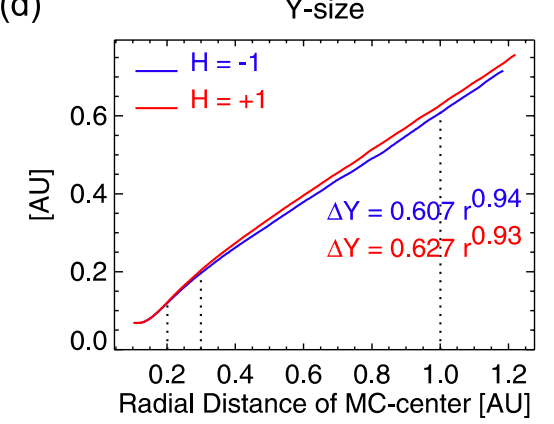

Fig. 8. Evolution of MC-boundaries and shock fronts as a function of radial distance to the Sun. In (a) the boundaries of $H_{\mathrm{e}}^{-}$(blue) and $H_{\mathrm{e}}^{+}$ (red) with regard to $x$ along $y=Y_{\mathrm{MC}-\text { center }}$ are presented. The maximum extent into y-direction is presented in (b). A comparison for the change of the clouds' sizes in $\mathrm{x}$ - and $\mathrm{y}$-directions is displayed in (c) and (d).

propagation, is displayed in Fig. 8d. It is arranged around $\sim 0.6 \mathrm{AU}$ for both magnetic clouds near Earth, which is about twice the extension in the $\mathrm{x}$-direction (see also a case study of Liu et al., 2008a). Figure 8c and 8d also contain exponential fits for the radial development of the size which are calculated for the radial range $0.3-1.0 \mathrm{AU}$. The base value given for the fit corresponds to $1 \mathrm{AU}$ distance from the Sun. The exponential value indicates the parameter's radial behavior. As can be seen from the fits, the evolution of extensions into y-direction is almost linear with radial distance (exponent close to 1), whereas the extension into x-direction follows an increase with $\sim r^{0.56}$.

More features concerning the positions and sizes of $H_{\mathrm{e}}^{-}$ and $H_{\mathrm{e}}^{+}$can be deduced from Fig. 9. The graphs in Fig. 9a and $9 \mathrm{e}$ compare the positions of both MC-centers. It can be seen that the center of $H_{\mathrm{e}}^{+}$is a little bit ahead of the center of $H_{\mathrm{e}}^{-}$at $1 \mathrm{AU}$ distance. The difference in radial direction is very small, of the order of $\sim 0.04 \mathrm{AU}$. A larger difference of $\sim 0.07 \mathrm{AU}$ is evident in y-direction indicating that the center of $H_{\mathrm{e}}^{-}$is drifting towards negative y-coordinates and that the center of $H_{\mathrm{e}}^{+}$is drifting towards positive y-coordinates. So, not only the cloud's boundaries are affected by magnetic reconnection with the IMF, but also the MC-centers are subject to a drift motion towards the site of magnetic reconnection.

Due to the fact that MC plasma is accelerated towards the reconnection site, it is obvious that propagation velocities for the centers of $H_{\mathrm{e}}^{-}$and $H_{\mathrm{e}}^{+}$are slightly different, as can be seen in Fig. 9b. Soon after initiation, the center-velocities decrease rapidly from the initial value of $800 \mathrm{~km} \mathrm{~s}^{-1}$ down to a value around $500 \mathrm{~km} \mathrm{~s}^{-1}$. This steep decrease is due to the hydrodynamic drag resulting from the slower ambient solar wind. When the MC-centers reach a distance of $\sim 0.3 \mathrm{AU}$, the velocity graphs start to level off, and deceleration becomes more moderate. Both center-velocities tend to approach the velocity of the background solar wind $\left(\sim 437 \mathrm{~km} \mathrm{~s}^{-1}\right.$ at $\left.1 \mathrm{AU}\right)$. The velocity for the center of $H_{\mathrm{e}}^{+}$ $\left(470 \mathrm{~km} \mathrm{~s}^{-1}\right)$ is slightly faster than for $H_{\mathrm{e}}^{-}\left(448 \mathrm{~km} \mathrm{~s}^{-1}\right)$ at $1 \mathrm{AU}$ because $H_{\mathrm{e}}^{+}$is accelerated towards the reconnection site lying ahead of the $\mathrm{MC}$, and $H_{\mathrm{e}}^{-}$is decelerated towards the reconnection site lying in the back.

Regarding the number density $n$ and thermal pressure $p_{\text {th }}$ given on the clouds' axes, there are nearly no differences visible after $63 \mathrm{~h}$ of propagation, as illustrated in Fig. 9c and 9d. After the phase of restructuring of the initial circular cross sections, $H_{\mathrm{e}}^{-}$and $H_{\mathrm{e}}^{+}$exhibit decays for $n$ and $p_{\text {th }}$ which are steeper than given in the ambient solar wind. Exponential fits within the range 0.3-1.0 AU yield a decay for $n$ with $r^{-2.58}$ and a decay for $p_{\text {th }}$ with $r^{-3.48}$. Corresponding relations in the ambient solar wind from the MHD simulations are $r^{-2.01}$ and $r^{-2.96}$, respectively. The decay of $n$ inside the magnetic cloud is in good agreement with values published for spacecraft observations (Bothmer and Schwenn, 1998; Liu et al., 
(a)

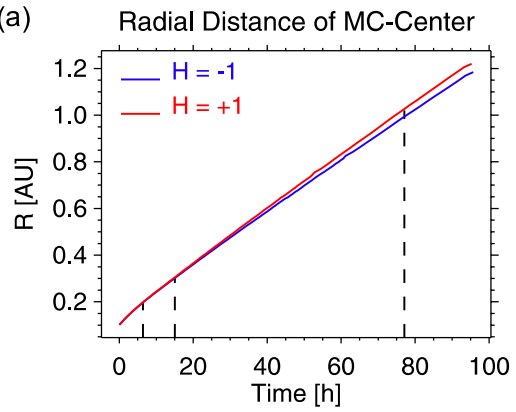

(b)

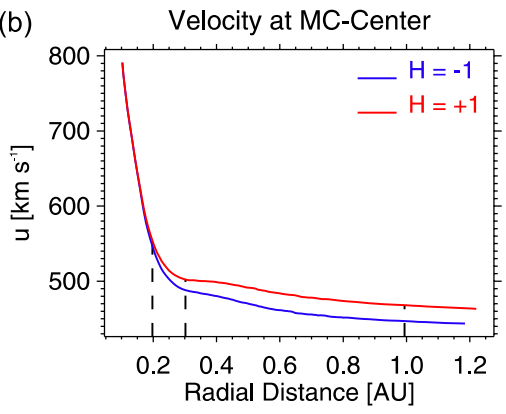

(c)
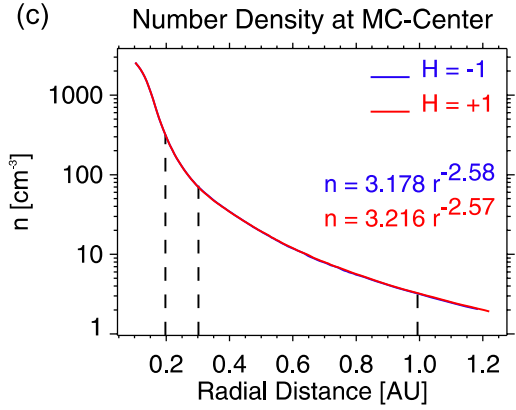

(d) Thermal Pressure at MC-Center

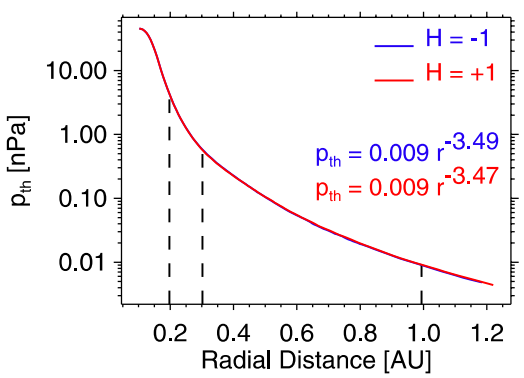

(e)

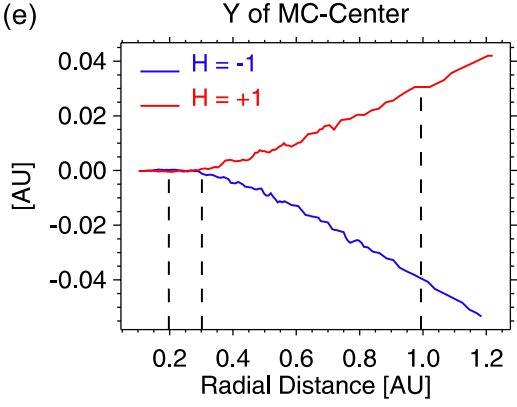

(f)

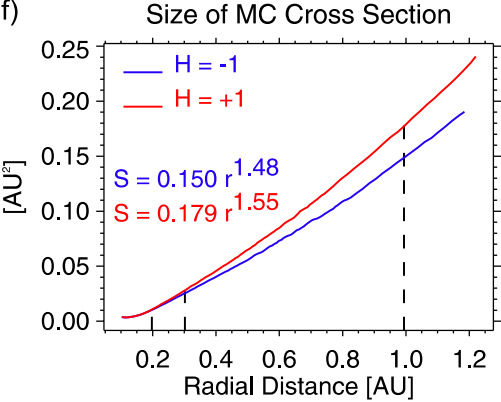

(g)

Mean Magnetic Field (Cross Section)

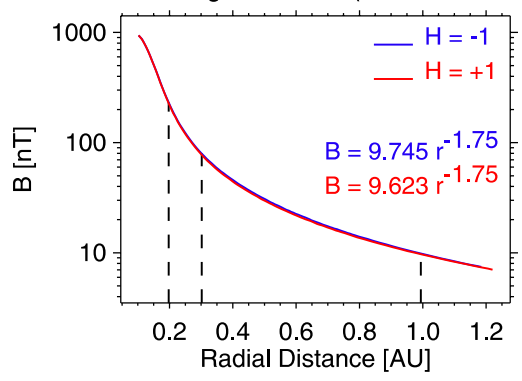

(h) Mean Force-Free Parameter (Cross Section)

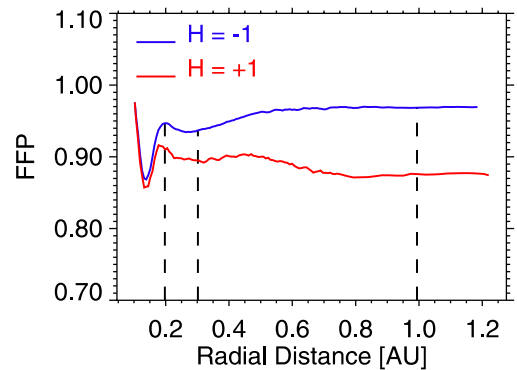

Fig. 9. Radial evolution of parameters at the MC-center and of parameters regarding the whole MC cross section. Graphs for $H_{\mathrm{e}}^{-}$are in blue, and graphs for $H_{\mathrm{e}}^{+}$are in red. Radial fits to simulation data between $0.3-1.0 \mathrm{AU}$ are listed as well.

2005, 2006; Wang et al., 2005). The radial decay of $p_{\text {th }}$ inside both MCs turns out to be a little bit too strong. A transformation from $p_{\text {th }}$ and $n$ into temperature $T$ by using the relation $p=n k T$, yields a decay for the temperature with $r^{-0.91}$. By contrast, exponential fits for $T$ taken from the literature (see above) are around $r^{-0.75}$. Smaller values for the polytropic index $\gamma$ would yield a more shallow decrease of $p_{\text {th }}$ and $T$ with radial distance. The MHD simulations are performed with a $\gamma=1.48$ which is set constant throughout the whole grid. Too strong cooling for the MC indicates that the magnetoplasma inside the cloud should be modeled with a smaller value for $\gamma$, e.g., $\gamma \sim 1.3$ (Liu et al., 2006). 

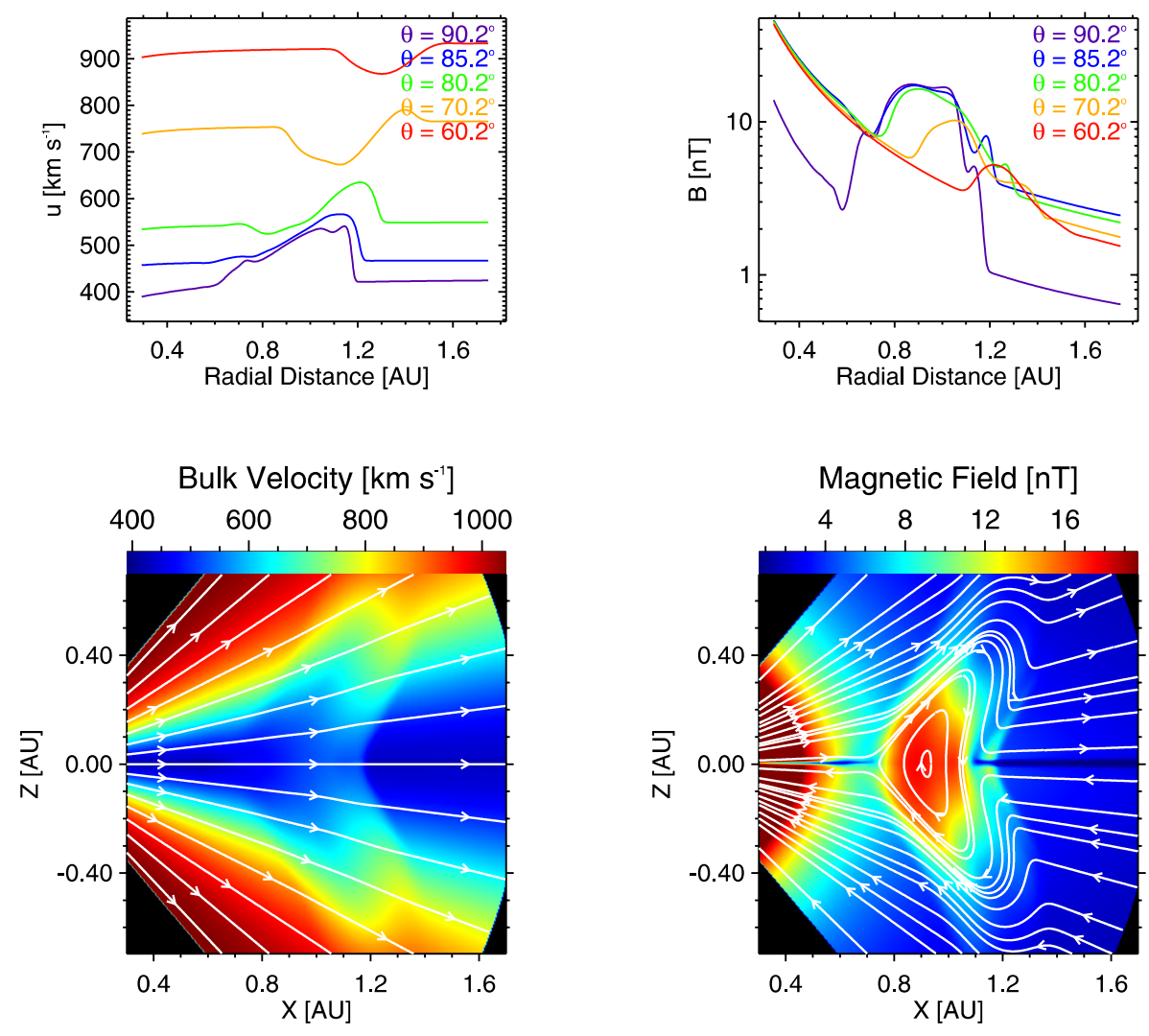

Fig. 10. Distributions for velocity (bottom left) and magnetic field (bottom right) for the $H_{m}^{-}$magnetic cloud at the distance of Earth (MCcenter at $\sim 0.91 \mathrm{AU})$. Corresponding radial profiles at constant polar angels are shown in the top row. Velocity streamlines and magnetic field lines are over-plotted in white color, respectively.

This takes into account additional heating mechanisms acting inside magnetic clouds like magnetic turbulence and local magnetic dissipation (Leamon et al., 1998). Such effects cannot be addressed by ideal MHD and are beyond the scope of this work.

The radial evolution of the sizes of 2-D cross sections is presented in Fig. 9f. $H_{\mathrm{e}}^{+}$develops a larger cross section than $H_{\mathrm{e}}^{-}$during propagation, which can again be attributed to magnetic reconnection with the IMF. It seems that magnetic reconnection on the front side is battling successfully against the process of MC-compression by the slower solar wind plasma ahead of the MC.

The radial development of the magnetic field for both clouds is illustrated by means of a mean magnetic field strength computed over the whole MC cross section. The mean magnetic field is a more representative value in this case than $B$ just on the cloud's axis, or the normal magnetic flux through the cross section. As can be seen in Fig. $9 \mathrm{~g}, H_{\mathrm{e}}^{-}$ and $H_{\mathrm{e}}^{+}$exhibit almost the same radial decay of their mean values for $B$. This decay is proportional to $r^{-1.75}$, which is exactly the same as that derived for the simulated ambient solar wind, and which is settled in between a $\sim r^{-1.64}$ from
Leitner et al. (2007) and $\sim r^{-1.85}$ from Gulisano et al. (2010) for the inner heliosphere.

Finally, a parameter for the degree of the force-free condition FFP of the cloud's magnetic field is presented in Fig. 9 h. Force-free means that $\nabla \times \boldsymbol{B}$ points into the direction of $\boldsymbol{B}$. Thus, the force-free parameter $F F P$ may be defined as the absolute value of the cosine of the angle between the two vectors $\boldsymbol{B}$ and $\nabla \times \boldsymbol{B}$, i.e.,

$$
F F P=|\cos (\boldsymbol{B}, \nabla \times \boldsymbol{B})|=\left|\frac{\boldsymbol{B} \cdot(\nabla \times \boldsymbol{B})}{|\boldsymbol{B}||\nabla \times \boldsymbol{B}|}\right| .
$$

Figure $9 \mathrm{~h}$ shows the radial development of the force-free parameter $F F P$ for both magnetic clouds, computed as a mean value over the whole $\mathrm{MC}$ cross section. The initial phase of restructuring of the circular cross section destroys the state of an exact force-free field given at the beginning. Nevertheless, FFP is again approaching high values close to 1 , especially for $H_{\mathrm{e}}^{-}$, while the MC is propagating away from the Sun $(F F P \sim 0.97$ at $1 \mathrm{AU})$. A lower $F F P \sim 0.88$ for $H_{\mathrm{e}}^{+}$at $1 \mathrm{AU}$ may be explained by a stronger deformation of the cloud's boundary, and thus, by a stronger deformation of the MC's magnetic field near the site of magnetic reconnection. It can be shown that $F F P$ adopts a value of exactly 1 

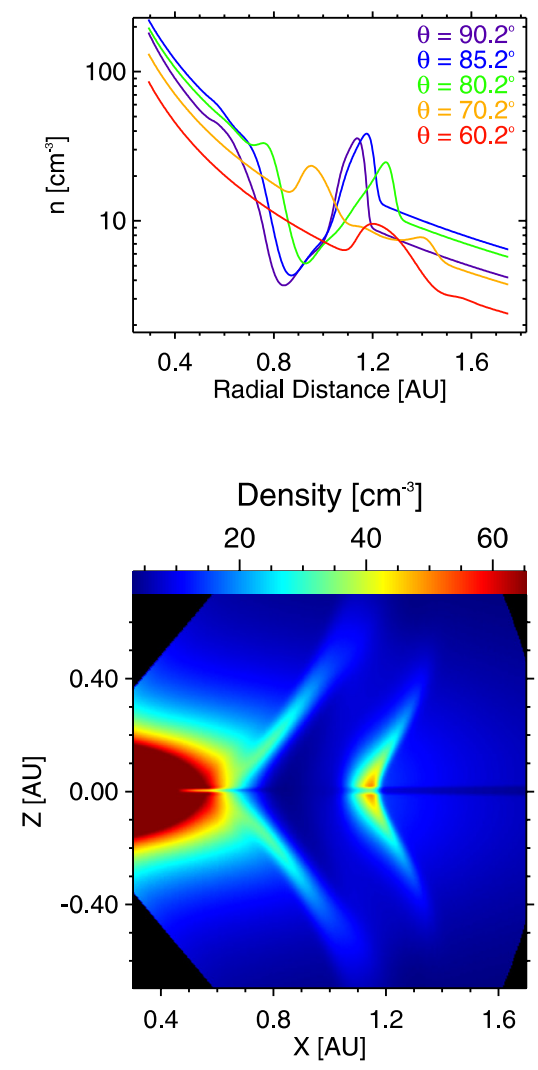

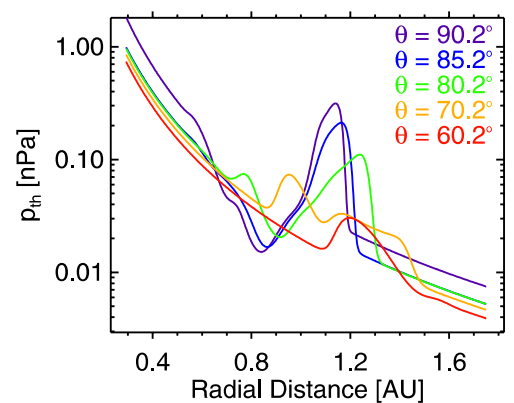

Thermal Pressure [nPa]

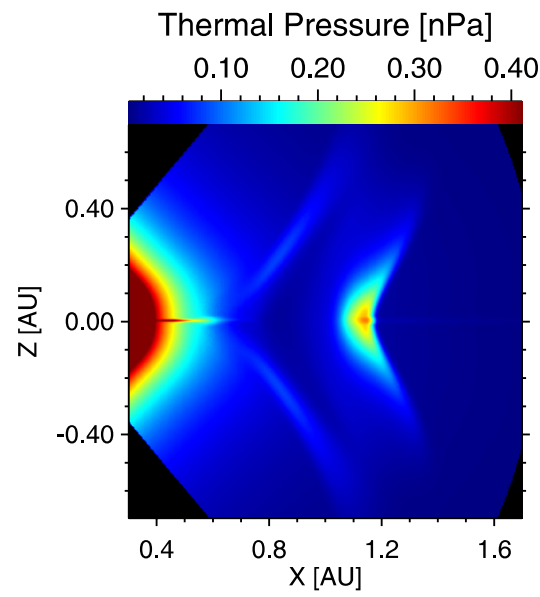

Fig. 11. Density (left) and thermal pressure (right) for the $H_{m}^{-}$magnetic cloud near Earth.

very quickly if it is not computed over the whole cross section but over a smaller area inside the MC, i.e., where the cloud's magnetic field lines are weakly influenced by ambient distortions (Möstl et al., 2009).

\section{Evolution of the MC cross section in the meridional plane}

In the second scenario, the propagation of an MC cross section in a meridional plane is the subject to 2.5-dimensional ideal MHD simulation. The ambient solar wind is highly structured with regard to latitude, and a heliospheric current sheet separates the two states of different radial $B_{\text {IMF }}$ orientations. Again, two types of magnetic clouds are released near the inner boundary with Lundquist force-free magnetic field configurations exhibiting a different magnetic handedness. For convenience, the expressions " $H_{m}^{-}$" and " $H_{m}^{+}$" are introduced to distinguish between those two cases of $H=-1$ and $H=+1$. The magnitude of the MCs' magnetic fields and the initial state of the plasmas are the same. Grids for the vector quantities $\boldsymbol{u}$ and $\boldsymbol{B}$ after $63 \mathrm{~h}$ of propagation are displayed in Fig. 10 and in Fig. 12. Parameters $n$ and $p_{\text {th }}$ are shown for the same time in Fig. 11 and in Fig. 13, when both magnetic clouds are arriving at Earth. The temporal evolutions of the two MCs' cross sections in the meridional plane reveal many similarities but also significant differences. They will be outlined in the following.

The two magnetic clouds are of the same initial size (circular cross section with radius $r_{\mathrm{mc}}=0.0375 \mathrm{AU}$ ), and they are launched from the same position near the inner boundary (MC-center at $x=0.1 \mathrm{AU}, z=0.0 \mathrm{AU}$ ), symmetrically with respect to the heliospheric current sheet. While moving away from the Sun, they experience strong expansion which is more efficient perpendicular to the direction of propagation as pointed out by Newkirk et al. (1981). Both magnetic clouds are seriously deformed from the initial circular shape into a "concave-outward" shape due to interaction with the structured ambient solar wind. This characteristic shape is often seen in MHD simulations (Manchester et al., 2004; Odstrcil et al., 2004) or may be concluded from spacecraft observations (Liu et al., 2008b). A flattened shape was also derived by Riley and Crooker (2004), who performed a pure kinematic treatment of MC propagation through a uniform solar wind. In a new analytical approach of force-free flux rope fitting, Démoulin and Dasso (2009) are taking into account the flattened/bent shape for the MC cross section by parameterizing its boundary. They found force-free solutions for non-circular cross sections and show that deviations from 

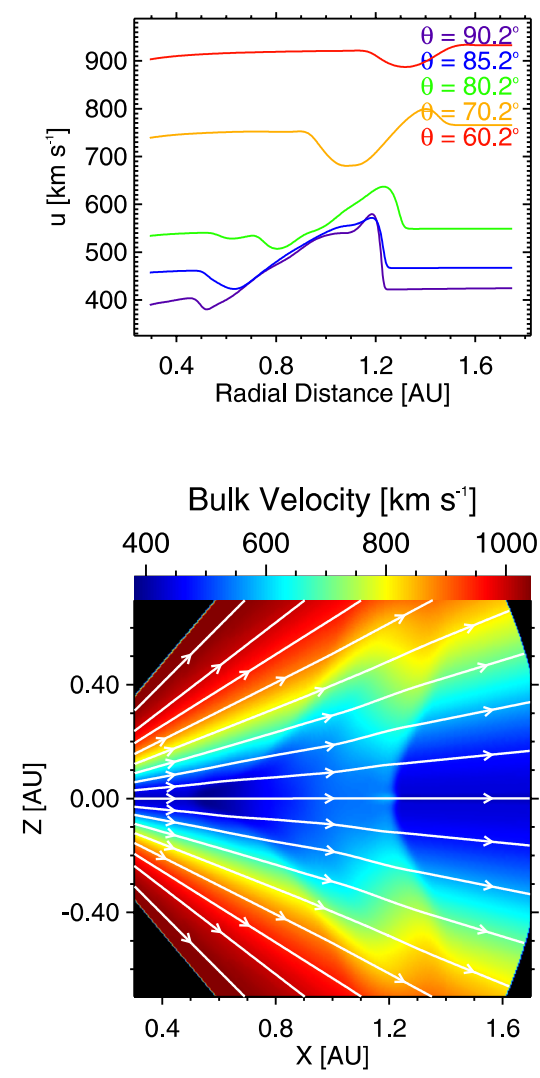
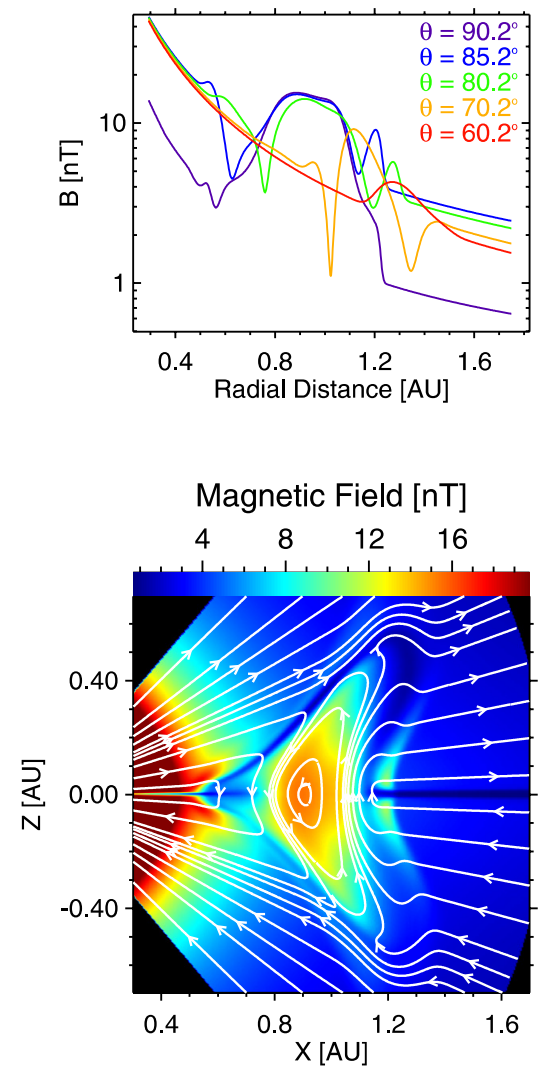

Fig. 12. Velocity (left) and magnetic field (right) for the $H_{m}^{+}$magnetic cloud near Earth (MC-center at $\left.\sim 0.90 \mathrm{AU}\right)$.

the standard circular shape mainly affect the distribution of magnetic field strength inside the MC, but not so its direction.

First, the deformation is evident from the evolution of the cloud's magnetic field lines. Figure 14a and b show the evolution of the outermost closed magnetic field line from a projection of 3-D field lines into the meridional plane. Time is depicted in rainbow colors on the field lines. These contours of magnetic field lines can be considered as the MC boundaries at different time steps. Expansion into the polar direction is much more pronounced than along the direction of propagation, i.e., the x-direction. Nevertheless, the shapes of $H_{m}^{-}$and $H_{m}^{+}$look different. While $H_{m}^{-}$develops an apex on the backside, $H_{m}^{+}$is more stretched into the polar direction. The extension into x-direction is larger for $H_{m}^{-}(0.31 \mathrm{AU})$ than for $H_{m}^{+}(0.26 \mathrm{AU})$, but the extension of $H_{m}^{+}$into z-direction $(0.92 \mathrm{AU})$ clearly exceeds that of $H_{m}^{-}$ $(0.70 \mathrm{AU})$. Values for the extensions are taken at $\sim 70 \mathrm{~h}$, when both MC-centers are located at $1 \mathrm{AU}$.

The hydrodynamic expansion velocity is illustrated in the lower row of Fig. 14 along the various paths of 180 selected test particles of the fluid. As already discussed for the equatorial scenario in Sect. 4, these particles are placed along a ring (fluid line) around the initial MC-center at the very first time step. As time evolves, particles begin to drift away from the MC-center. The expansion velocity $\boldsymbol{u}_{\text {exp }}$, i.e., the fluid velocity of the test particles relative to the velocity of the MC-center, is sketched in Fig. $14 \mathrm{c}$ for $H_{m}^{-}$and in Fig. 14d for $H_{m}^{+}$. Two peaks into positive and negative z-directions near the MC-center are visible (red-yellow shades). Soon after launch, both magnetic clouds expand heavily with a maximum amplitude of $\boldsymbol{u}_{\text {exp }} \approx 190 \mathrm{~km} \mathrm{~s}^{-1}$. After approximately $20 \mathrm{~h}, \boldsymbol{u}_{\text {exp }}$ slows down to $110-120 \mathrm{~km} \mathrm{~s}^{-1}$ in the vertical direction and to $<50 \mathrm{~km} \mathrm{~s}^{-1}$ in the $\mathrm{x}$-direction. The fluid lines are drawn every $5 \mathrm{~h}$ as gray contours.

Due to expansion, the interiors of both MCs become filled with a low- $\beta$ plasma. Furthermore, the supersonic flow creates a fast mode shock and a sheath ahead of $H_{m}^{-}$and $H_{m}^{+}$. This is clearly visible in the enhancements of $n$ and $p_{\text {th }}$ in Fig. 11 and in Fig. 13. The shock stand-off distance becomes a function of latitude, and it is smaller near the equatorial plane. Furthermore, there is a post-shock compression visible on the backside as well.

A comparison of shapes is also illustrated in Fig. 15. Figure 15a displays the evolution of the backside, the front boundary, and the shock front for both magnetic clouds along the $\mathrm{x}$-direction at $z=Z_{\mathrm{MC}-\text { center }} \approx 0$. Distances are normalized to the $\mathrm{x}$-distance of the MC-center. As already noted, 

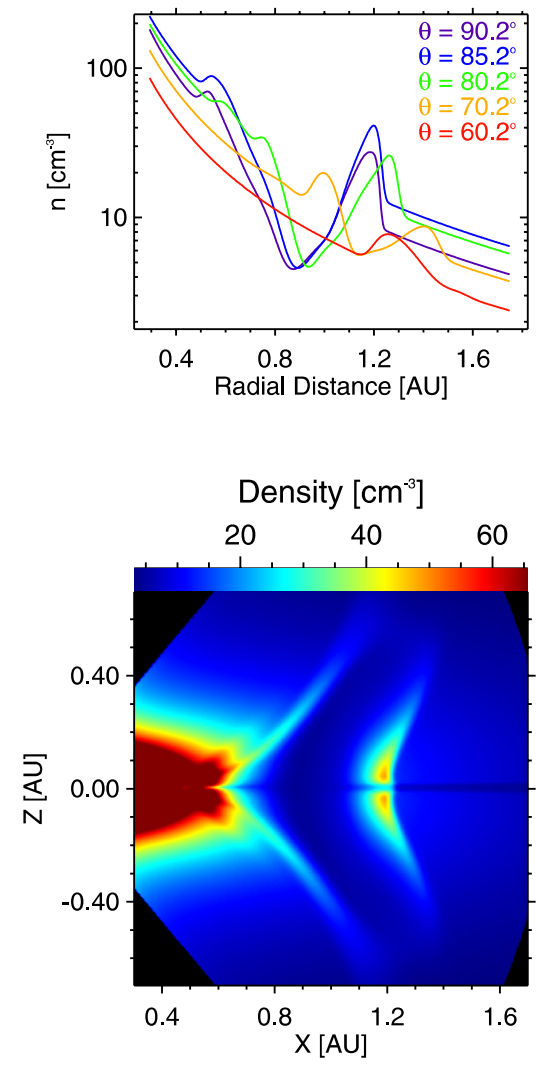
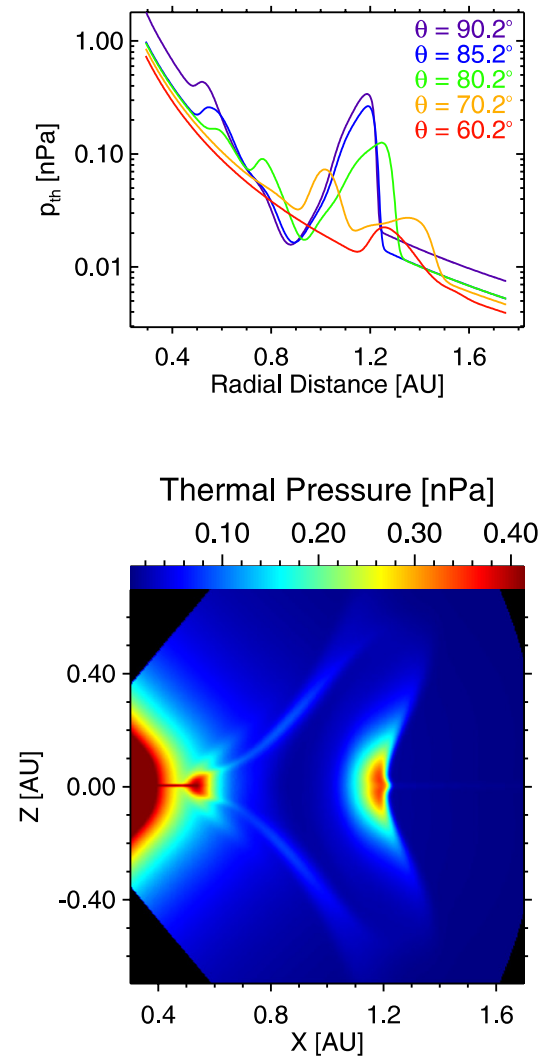

Fig. 13. Density (left) and thermal pressure (right) for the $H_{m}^{+}$magnetic cloud near Earth.

$H_{m}^{-}$exhibits a larger extension towards the backside. On the other hand, $H_{m}^{+}$develops a larger extension into z-direction, as can be seen in Fig. 15b and 15d. Furthermore, radial fits computed for the range $0.3-1.0 \mathrm{AU}$ are listed.

Figure 15a includes the evolution of the shock stand-off distance near the equatorial plane as a function of radial distance to the Sun. It is clearly larger for $H_{m}^{+}(\sim 0.22 \mathrm{AU})$ than for $H_{m}^{-}(\sim 0.17 \mathrm{AU})$ at $1 \mathrm{AU}$. This is mainly caused by the different geometries of magnetic field lines inside the sheath region. For $H_{m}^{-}$, IMF field lines are always turned around the $\mathrm{MC}$, whereas for $\mathrm{H}_{m}^{+}$, they are bent towards the equator and become reconnected across the equator. In front of $H_{m}^{+}$, magnetic reconnection of IMF field lines along a broad range inside its sheath seems to provoke additional acceleration of plasma into positive $\mathrm{x}$-direction. Thus, the shock front is pushed a little bit further ahead of the cloud, and the shock stand-off distance is increasing. Behind $\mathrm{H}_{m}^{+}$, magnetic field lines become reconnected across the equator as well. Care should be taken that this "tail" of field lines exhibits the same orientation as the cloud's backside magnetic field, but it already belongs to the IMF and not to the cloud anymore.

The influence of the cloud's magnetic handedness on the orientation of the IMF ahead of the cloud is of special importance for triggering geomagnetic storms at Earth (Dungey,
1961; Farrugia et al., 1994, 1997; Biernat et al., 2000). $H_{m}^{-}$ forces the IMF to become oriented in north-south direction inside its sheath. On the contrary, the IMF inside the sheath of $H_{m}^{+}$becomes oriented in south-north direction. So, the sheath magnetic field of $H_{m}^{-}$is directed oppositely to the Earth's dayside magnetic field, which in turn favors magnetic reconnection at the terrestrial magnetopause. Thus, not only $H_{m}^{-}$itself but also its sheath will be more geo-effective than $H_{m}^{+}$.

Two other outstanding regions for magnetic reconnection are located at the rear flanks of $H_{m}^{+}$in the Northern and Southern Hemispheres, where magnetic field lines of the cloud and the IMF are antiparallel (see magnetic field lines in Fig. 12 around $x \approx 1.04 \mathrm{AU}, z \approx \pm 0.42 \mathrm{AU}$ ). Thus, it is expected that $H_{m}^{+}$may lose some amount of magnetic flux to the IMF while propagating through the heliosphere. This will be discussed in more detail later on.

In principle, the plasma velocities point in the radial direction away from the Sun, with magnitudes larger than $400 \mathrm{~km} \mathrm{~s}^{-1}$. The other two spherical velocity components $u_{\theta}$ and $u_{\phi}(\theta$ is polar distance, $\phi$ is azimuth) play a minor role with magnitudes usually less than $50 \mathrm{~km} \mathrm{~s}^{-1}$. Nevertheless, it is interesting to focus on some peculiarities of the meridional flow pattern. Therefore, the grids of the velocity 

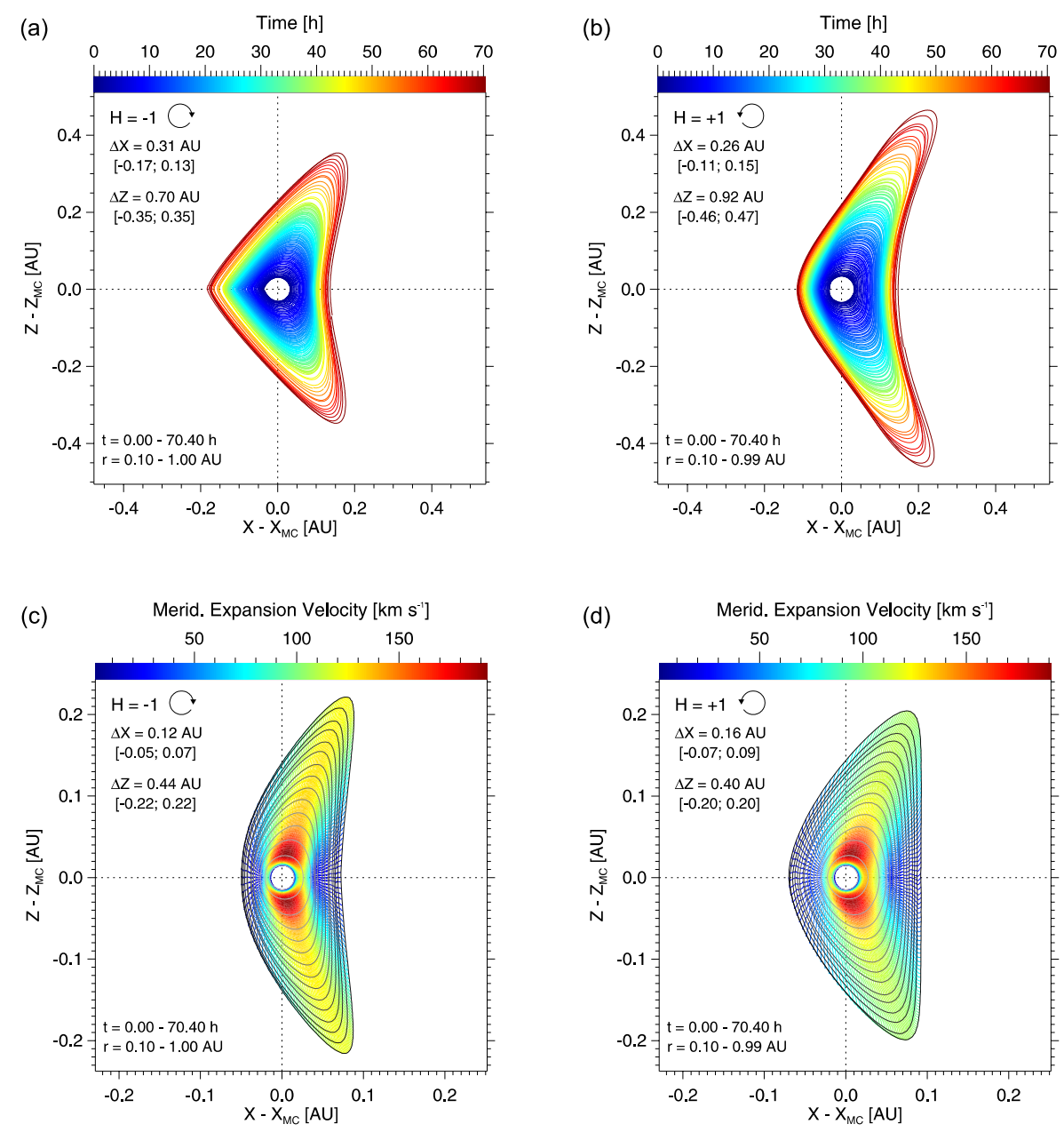

Fig. 14. The outermost "closed" magnetic field line as a function of time for $H_{m}^{-}$(a) and $H_{m}^{+}$(b). The lower row presents the hydrodynamic expansion velocities along the various paths of 180 test particles inside $H_{m}^{-}$(c) and inside $H_{m}^{+}$(d) (see text). Gray contours are drawn every $5 \mathrm{~h}$.

components $u_{\mathrm{r}}$ and $u_{\theta}$ are displayed in Fig. 16 for $H_{m}^{-}$(see $16 \mathrm{a}$ and 16b) and for $H_{m}^{+}$(see 16d and 16e). Magnetic field lines are over-plotted in gray or white color in order to be able to estimate the position of the $\mathrm{MC}$ with regard to the ambient solar wind. Inside the sheath regions of both MCs, the velocity component $u_{\theta}$ mainly points towards higher latitudes. While the slower solar wind plasma is overtaken and compressed by the MC, it is deflected around the MC body towards higher northern and southern latitudes. Partially, this is also true for the backside, where fast solar wind flows at higher latitudes ram into the cloud and are deflected towards the poles as well. Nevertheless, there is a narrow strip visible in the grid for $u_{\theta}$ along the foreshock exhibiting exactly the opposite behavior. Immediately behind the shock front inside the sheath, $u_{\theta}$ first points towards the equator, with amplitudes $\leq 20 \mathrm{~km} \mathrm{~s}^{-1}$ (see positive $u_{\theta}$ (red) in the Northern Hemisphere, and negative $u_{\theta}$ (blue) in the Southern Hemisphere). So, the shocked solar wind plasma is first deflected a little bit towards the equator before it is passed around the MC towards higher latitudes. Manchester et al. (2005) have clearly demonstrated in the frame of MHD simulations that a concave-outward shape of a fast mode shock causes both, a bending of the flow and of magnetic field lines away from the shock normal (in this particular case towards the equator). Liu et al. (2008b) analyzed ICME data recorded by the Wind satellite and have been able to identify equatorward flows ahead of ICMEs, too. These flows lead to a stronger compression and heating of plasma in the equatorial sheath. A closer inspection of the $B_{\theta}$-grids in Fig. $16 \mathrm{c}$ and $16 \mathrm{f}$ reveals the bending of IMF field lines away from the shock normal, i.e., towards the equator, immediately behind the shock front. Thus, $B_{\theta}$ at the shock turns out to be always weakly negative (blue). Deeper inside the sheath, $B_{\theta}$ becomes dominantly positive (red) for $H_{m}^{-}$, indicating that $\boldsymbol{B}$ is turned around the MC. For $H_{m}^{+}, B_{\theta}$ first switches from negative at the shock to positive deeper inside the sheath, 
(a)

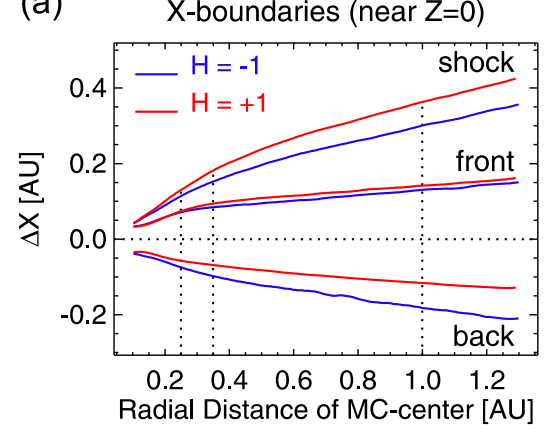

(c)

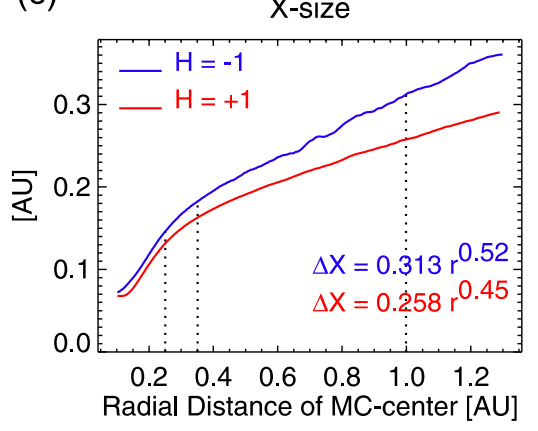

(b)

\section{Z-boundaries}

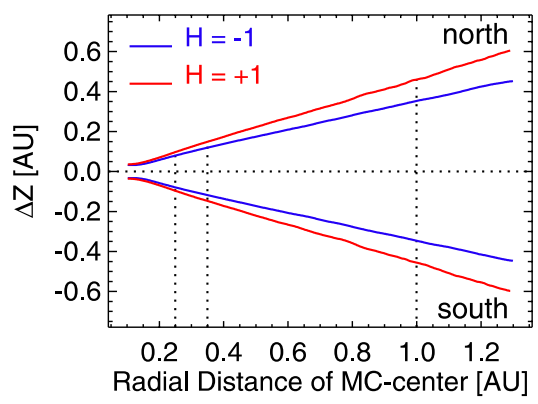

(d)

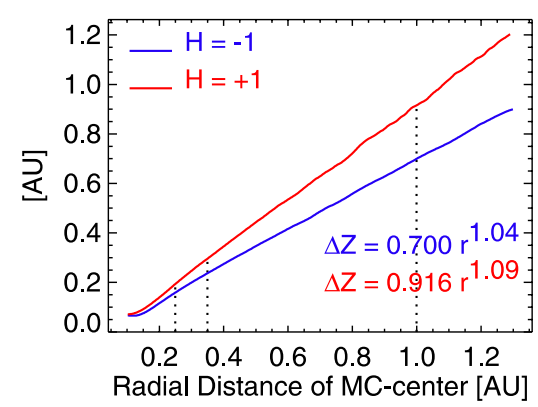

Fig. 15. Evolution of the MC-boundaries and shock fronts for $H_{m}^{-}$(in blue) and $H_{m}^{+}$(in red) (a, b). Boundary and shock positions are taken

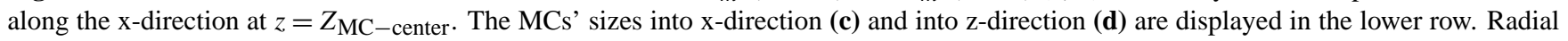
fits inside the range $0.3-1.0 \mathrm{AU}$ are listed as well.

and then again switches sign so that IMF field lines finally become reconnected across the equator. Exactly in the equatorial plane, the equatorward flows from both hemispheres cause a collision of IMF field lines belonging to opposite orientations. This boosts magnetic reconnection directly inside the equatorial plane at the foreshock, not only for $H_{m}^{+}$but also for $H_{m}^{-}$. The point of magnetic reconnection is characterized by merging magnetic field lines, decreased magnetic field strength, decreased plasma density, increased thermal pressure, and an increased plasma velocity. Plasma is accelerated away from the reconnection site along the radial direction, nearly parallel to the magnetic field, resulting in a small peak for $u_{\mathrm{r}}$ at $z=0, x \sim 1.16 \mathrm{AU}$, and a small sink for $u_{\mathrm{r}}$ at $z=0, x \sim 1.10 \mathrm{AU}$ (see Fig. 16a and 16d). The sink is due to a superposition of the shocked solar wind flow with a reconnection jet pointing into negative $\mathrm{x}$-direction. The peak results from a positive superposition of the shocked solar wind flow with a reconnection jet into positive x-direction. As a consequence, the shock front is pushed further downstream of the MC, thereby increasing somewhat the shock standoff distance. This so-called "pimple" seen in the equatorial shock front of MHD simulations has been reported earlier by various authors (see, e.g., Burton et al., 1992; Odstrčil et al., 1996, or Manchester et al., 2005). Uralova and Uralov (1994) first suggested that a small velocity component towards the
HCS is able to initiate the magnetic reconnection process accompanying a shock wave.

Several other parameters concerning the centers and cross sections of both MCs are summarized in Fig. 17. Figure 17a and $17 \mathrm{~b}$ show a comparison of time-height profiles and speed profiles. As can be seen, the centers of both clouds arrive at the same time $(\sim 70 \mathrm{~h})$ at $1 \mathrm{AU}$ distance. Nevertheless, $H_{m}^{+}$ would be detected earlier at Earth by the arrival of its shock front due to a larger shock stand-off distance. The speed profiles of both clouds shown in Fig. 17b reveal nearly the same behavior. Soon after launch, both clouds are heavily decelerated from the initial speed of $800 \mathrm{~km} \mathrm{~s}^{-1}$ down to $554 \mathrm{~km} \mathrm{~s}^{-1}$ $\left(H_{m}^{-}\right)$and $529 \mathrm{~km} \mathrm{~s}^{-1}\left(H_{m}^{+}\right)$at $0.25 \mathrm{AU}$, respectively. So, both clouds lose about $1 / 3$ of their initial speed during the first $0.15 \mathrm{AU}$ of propagation. After a small increase of velocities between $0.25-0.35 \mathrm{AU}, H_{m}^{-}$and $H_{m}^{+}$are again moderately decelerated approaching a velocity of $\sim 500 \mathrm{~km} \mathrm{~s}^{-1}$ at $1 \mathrm{AU}$. A kink in both velocity profiles at $0.25 \mathrm{AU}$ may be rather a result of restructuring of the cloud's interior due to the MHD-violating initial conditions. One has to bear in mind that the initial MC cross section is just a circular part of the solar wind grid, which is filled ad hoc with a modeled state for the plasma and for the magnetic field. No special boundary conditions are specified for the transition region between the MC and the ambient solar wind. This violates 

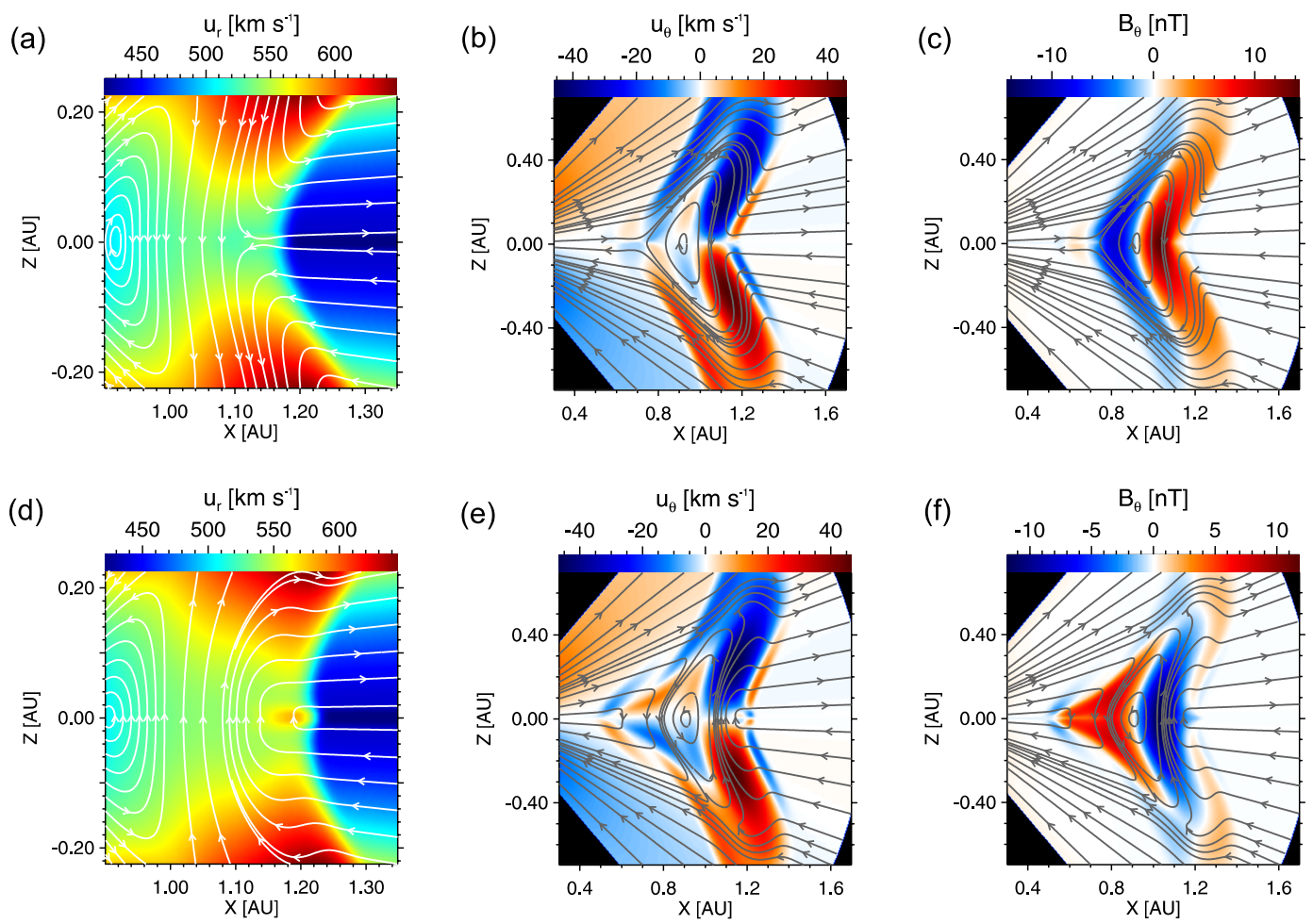

Fig. 16. Grids for the spherical velocity components $u_{\mathrm{r}}$ and $u_{\theta}$ of $H_{m}^{-}$(a, b) and $H_{m}^{+}$(d, e). Grids (a) and (d) are zooming into the equatorial shock front. The polar magnetic field $B_{\theta}$ is displayed in (c) for $H_{m}^{-}$and in (f) for $H_{m}^{+}$. Magnetic field lines are over-plotted on each grid.

the laws of MHD, but after several hundred time steps, numerical diffusion acts in a way so that the solution becomes again physically reliable.

Figure $17 \mathrm{c}$ and $17 \mathrm{~d}$ show the change of number density $n$ and thermal pressure $p_{\text {th }}$ at the MC-center. There is a steep decrease visible in both parameters during the first few hours of propagation up to a distance of $0.25 \mathrm{AU}$. This indicates strong depletion and cooling of plasma at the center during the initial phase of rapid expansion. Later on, the profiles of $n$ and $p_{\text {th }}$ pass into a more shallow decrease. Differences are only visible regarding $p_{\text {th }}$, which turns out to be a little bit higher for $H_{m}^{-}$than for $H_{m}^{+}$. Radial fits performed inside the range $0.3-1.0 \mathrm{AU}$ reveal a stronger decrease of $n$ and $p_{\text {th }}$ inside the magnetic clouds than given in the ambient solar wind. As already discussed for the equatorial scenario in Sect. 4, the cloud's interior should be modeled with a lower polytropic index, e.g., $\gamma \sim 1.3$, to take into account additional heating of the MC plasma (Liu et al., 2006).

The evolution of the clouds' magnetic fields is illustrated by means of two parameters: First, by the mean magnetic field strength computed over the whole MC cross section (see Fig. 17f), and second, by the absolute value of the normal magnetic flux $\Psi$ through the cross sectional area (see Fig. 17g). By definition, the MC cross section is confined by the outermost 2-D projected closed magnetic field line encircling the MC's center. The radial evolution of its size $S$ is depicted in Fig. 17e. Deformation of the cloud's cross section due to interaction with the ambient solar wind leads to a larger cross section for $H_{m}^{+}$than for $H_{m}^{-}$. As can be seen from Fig. 17f, $H_{m}^{+}$arrives with a lower mean magnetic field at Earth than $H_{m}^{-}$. This may be explained by the fact that the magnetic field of $H_{m}^{+}$is strongly reconnecting to the IMF thereby losing magnetic flux to the IMF. For $H_{m}^{-}$, magnetic reconnection with the IMF plays a minor role. Thus, the cloud's magnetic flux is conserved much better.

The magnetic flux $\Psi$ through a surface $S$ is defined as

$\Psi=\int \boldsymbol{B} \cdot \boldsymbol{n} \mathrm{dS} \quad[\mathrm{Mx}]$,

and it is given in the unit Maxwell $\left([\mathrm{Mx}]=10^{8} \times\left[\mathrm{T} \mathrm{m}^{2}\right]\right)$. The component oriented normal to the cross section in the meridional propagation scenario corresponds to $B_{\phi}$. Despite the fact that the cross section of $H_{m}^{+}$is larger than that of $H_{m}^{-}$, the former exhibits a lower magnetic flux at $1 \mathrm{AU}$ (see Fig. $17 \mathrm{~g}$ ). This is again a clear indication that more magnetic flux of $H_{m}^{+}$gets lost to the IMF by magnetic reconnection, especially at the rear flanks, where magnetic field orientations of $H_{m}^{+}$and the IMF are antiparallel (see also magnetic field lines in Fig. 12).

Between the inner boundary of the grid and a distance of $1 \mathrm{AU}$ to the Sun, approximately $35 \%-40 \%$ of the original 
(a)

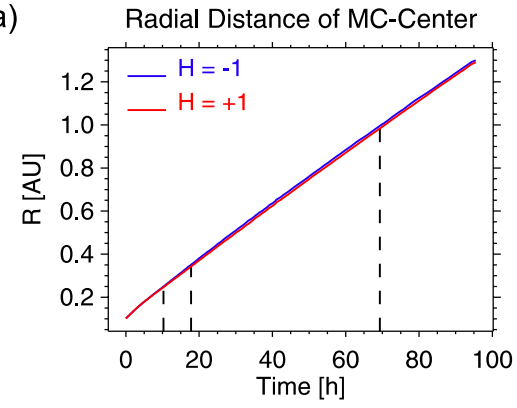

(b)

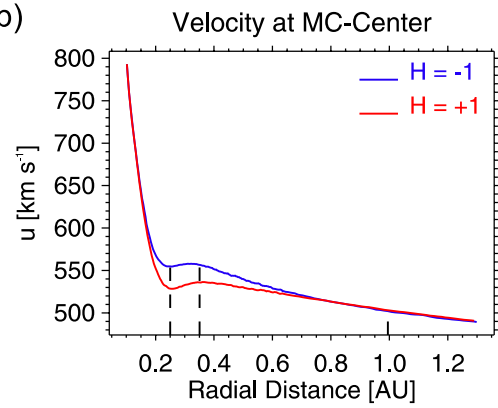

(c)

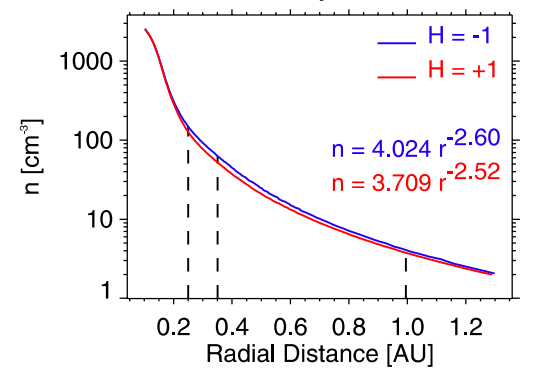

(d) Thermal Pressure at MC-Center

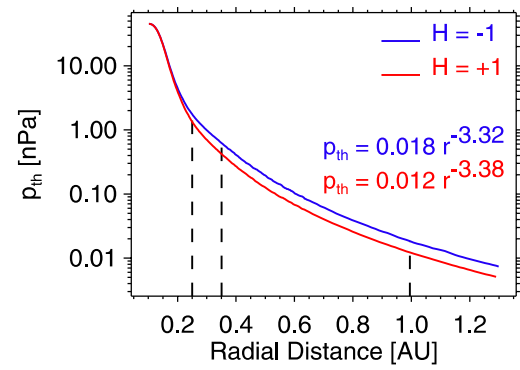

(e) Size of MC Cross Section

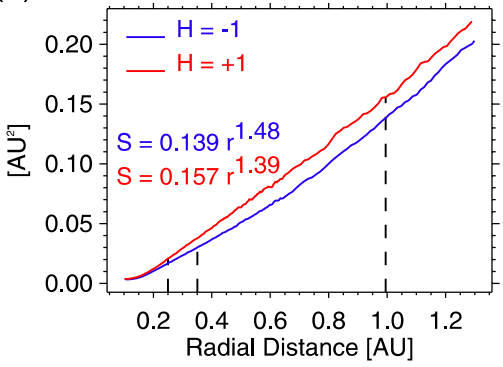

(f) Mean Magnetic Field (Cross Section)

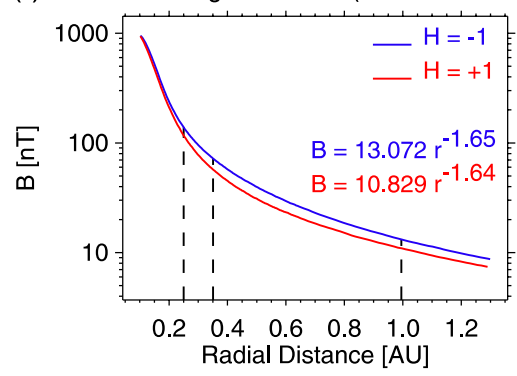

(g) Magnetic Flux through Cross Section

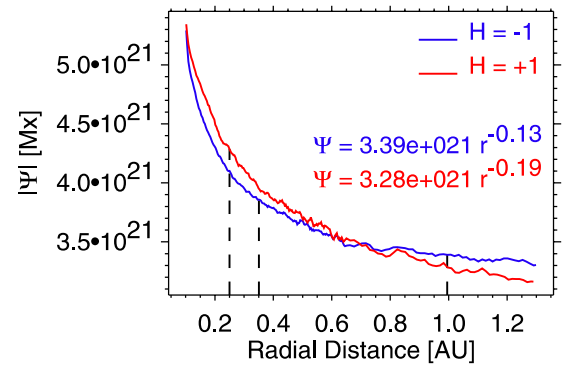

(h) Mean Force-Free Parameter (Cross Section)

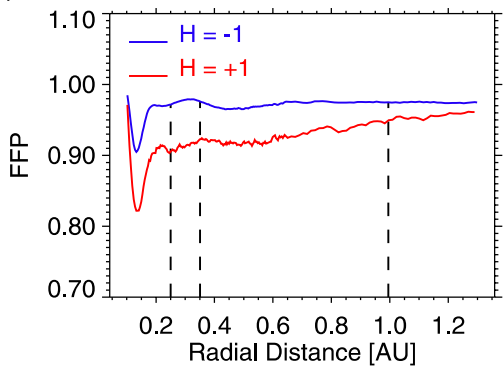

Fig. 17. Radial evolution of parameters at the MC-center (left column) and of parameters which are computed over the whole MC cross section (right column) for $H_{m}^{-}$(blue) and $H_{m}^{+}$(red). Radial fits computed for the range $0.3-1.0 \mathrm{AU}$ are listed where meaningful.

flux gets lost for both MCs. Dasso et al. $(2006,2007)$ attribute the loss of magnetic flux $(20 \%-30 \%)$ to a "peeling" of the flux rope on its way to Earth. According to the slopes of $\Psi$ shown in Fig. 17g, this kind of "peeling" of the flux rope is stronger near the Sun than farther away. The possibility of an even higher amount of reconnected flux has been demonstrated by Möstl et al. (2008), who concluded a loss of $\sim 50 \%$ for a single magnetic cloud event.
Furthermore, it is interesting to note that due to the deformation of the $\mathrm{MC}$, the point of maximum magnetic field strength is not exactly placed on the axis anymore, but it is placed a little bit behind the axis, as has already been the case for the equatorial propagation scenario.

Finally, the radial development of the mean force-free parameter $F F P$ computed over the cross sections of $H_{m}^{-}$and $H_{m}^{+}$is presented in Fig. 17h. FFP is defined in Eq. (6), 

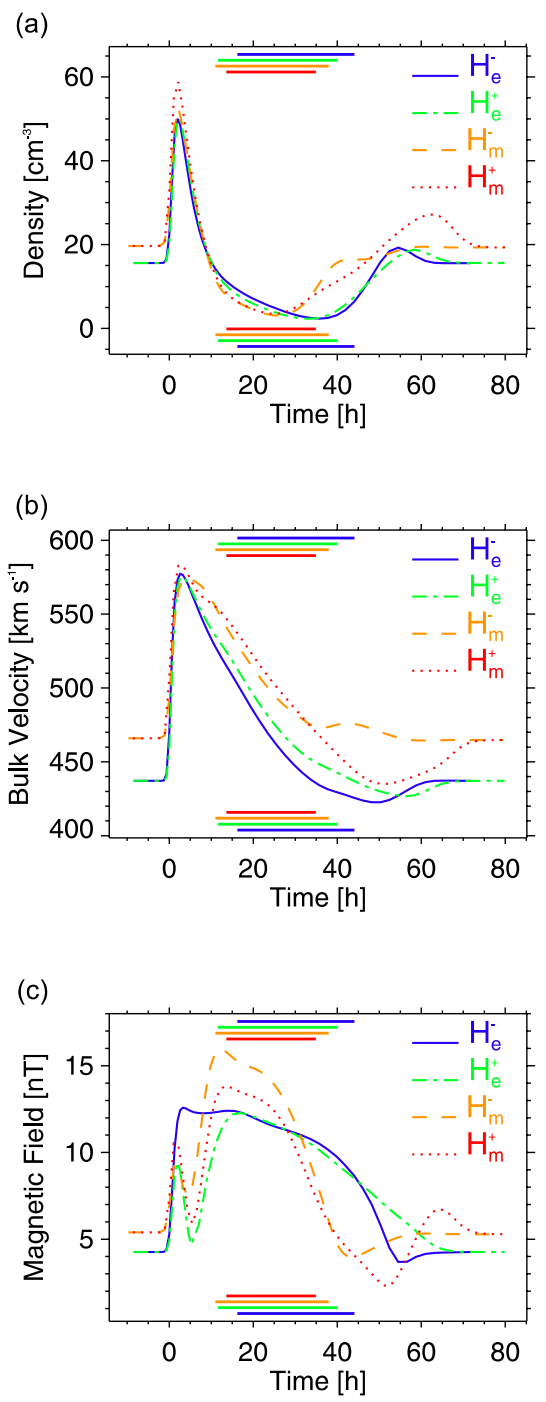
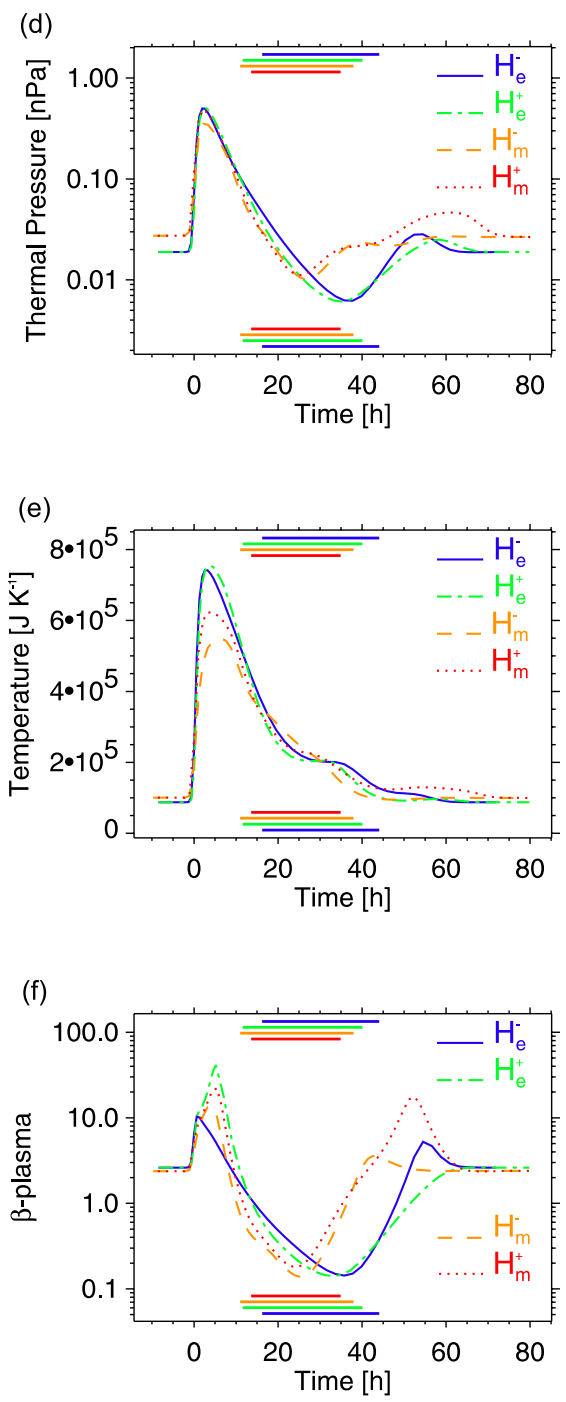

Fig. 18. Number density (a), bulk velocity (b), magnetic field strength (c), thermal pressure (d), temperature (e), and plasma- $\beta$ (f) measured by a virtual spacecraft at $1 \mathrm{AU}$ near the equatorial plane. Profiles of the equatorial propagation scenarios $\left(H_{\mathrm{e}}^{-}, H_{\mathrm{e}}^{+}\right)$and of the meridional propagation scenarios $\left(H_{m}^{-}, H_{m}^{+}\right)$are all superposed. Shock times are synchronized to $0.0 \mathrm{~h}$. Horizontal bars indicate the MC's interior which is, by definition, bounded by the outermost closed magnetic field line encircling the center.

and it is expressing the cosine of the angle between the two vectors $\nabla \times \boldsymbol{B}$ and $\boldsymbol{B}$. The initial exact force-free configuration is destroyed for both clouds soon after launch from the inner boundary due to strong expansion and restructuring (see drop of $F F P$ near $0.13 \mathrm{AU}$ ). Later on, $F F P$ is going to approach the value of 1 again while the MC is propagating away from the Sun. At $1 \mathrm{AU}, H_{m}^{-}$exhibits an $F F P \sim 0.97$, which is slightly higher than $F F P \sim 0.95$ for $H_{m}^{+}$. This is not surprising because $H_{m}^{+}$reconnects extensively to the IMF thereby lowering $F F P$, especially in regions close to the reconnection sites. Nevertheless, it seems that an initial force-free magnetic field configuration is very well preserved inside magnetic clouds. Even after strong deviation from the force-free configuration, it is recaptured again and seems to be a favored state which is approximated automatically (at least in ideal MHD).

\section{Conclusions}

The propagation of magnetic clouds through the inner heliosphere $(0.05-1.75 \mathrm{AU})$ has been investigated using 2.5dimensional numerical MHD simulations. A Roe-type approximate Riemann solver (Godunov 1959; Roe, 1981) has been constructed on the basis of eigenvalues and eigenvectors for the linearized system (Powell, 1994). An MHD solution for the background plasma yields plausible conditions 
for a quiet solar wind during a solar minimum phase. The initial magnetic cloud is modeled as a 2-D circular cross section with a constant- $\alpha$ force-free magnetic field (Lundquist, 1950; Burlaga, 1988). Four different scenarios of initial MC configurations have been simulated with special emphasis placed on the role of the initial magnetic handedness. This parameter strongly influences the efficiency of magnetic reconnection between the MC's magnetic field and the interplanetary magnetic field. All four propagation scenarios, i.e., the equatorial and the meridional ones, comprise the following common features: Strong expansion during propagation, low values for the plasma- $\beta$ inside the MC, deceleration towards the speed of the ambient solar wind, a fast mode shock and a sheath ahead of the MC, and strong deformation of the initial circular cross section.

The propagation of an MC with its axis oriented perpendicular to the equatorial plane leads to an elliptic shape. Expansion is stronger perpendicular to the direction of propagation, i.e., into y-direction. Furthermore, the ellipses' semimajor axes are tilted with regard to the y-direction, and the whole cross section is drifting along the y-direction. The tilt of the ellipse is a new feature seen in this work. It is caused by a deceleration of plasma in the radial direction close to the reconnection site. On the other hand, the drift motion is caused by an acceleration of MC plasma in the azimuthal direction towards the reconnection site. Depending on the MC's handedness, magnetic reconnection between the MC and the Parker spiral IMF either occurs on the front side or on the backside. Such a drift of MC plasma towards the reconnection site has already been reported by Vandas et al. $(1995,1996)$. They derived an azimuthal offset for the MC-center from the $\mathrm{x}$-axis of $\sim 0.1 \mathrm{AU}$ at $1 \mathrm{AU}$ distance from the Sun. This is much more than the $\sim 0.04 \mathrm{AU}$ calculated in this work. The difference may be explained by different numerical solvers, different grid resolutions, and thus, different numerical diffusions regulating the intensity of magnetic reconnection.

An extrapolation of these 2.5-D results to a fully 3-D scenario allows to make the following considerations. In full $3-\mathrm{D}$, the radial component of the IMF changes sign over the equator, and a tilt between the Sun's rotation axis and its magnetic axis creates a wavy current sheet. So, if the axis of the flux rope is locally oriented perpendicular to the equatorial plane, the signatures of both cases of opposite magnetic handedness discussed above will be observed at the same time. In one hemisphere, the site of magnetic reconnection will be ahead of the flux rope, and in the other hemisphere, it will be on the backside. This leads to a distortion of the flux rope depending on hemisphere. The 2.5-D studies presented here may serve as a basis under more simple geometrical conditions to understand more complicated effects seen in 3-D simulations.

An orientation of the MC's axis parallel to the equatorial plane leads to a concave-outward shape for the meridional cross section during propagation in a bi-modal ambient solar wind typical of solar activity minimum. The initial magnetic handedness of the MC determines how the MC starts to interact with the interplanetary magnetic field. This is of vital importance especially at the front side shock and sheath because it determines the orientation of the sheath's magnetic field, and thus influences the MC's geo-effectiveness at Earth. Depending on the handedness, IMF field lines are either turned around the MC body or they become reconnected across the heliospheric current sheet. Furthermore, there is significant magnetic reconnection visible between the MC and the IMF along the rear flanks of $H_{m}^{+}$where magnetic field orientations are antiparallel. Such studies have already been performed by Cargill and Schmidt (2002), and Schmidt and Cargill (2003) on the basis of a uniform ambient solar wind. The present work provides a more realistic background solar wind designed especially for solar minimum conditions with density, velocity, magnetic field, and pressure depending on heliographic latitude. Magnetic reconnection between a magnetic cloud and the IMF under such conditions has never been reported before.

The presence of an equatorward flow of plasma showing up in front of concave-outward shaped magnetic clouds could be confirmed. Such flows have been predicted by Burton et al. (1992), simulated by Manchester et al. (2005), and they have been detected by Liu et al. (2008b) in ACE and Wind observations. This work shows that equatorward flows develop independently of the magnetic handedness of the $\mathrm{MC}$, and they seem to be a direct consequence of the shape of the cloud's shock front. The maximum amplitude derived for these flows is rather low $\left(<20 \mathrm{~km} \mathrm{~s}^{-1}\right)$, but would increase with increasing curvature for the MC and its front-side shock. The curvature itself depends on the relative speeds between the MC and the solar wind as a function of heliographic latitude. Furthermore, our MHD simulations revealed that these equatorward flows can boost the process of magnetic reconnection of IMF field lines in front of the MC.

Finally, the issue of force-free magnetic fields is also addressed by this study. The degree of force-freeness is parameterized, and its evolution is pursued during propagation of the MC from the inner boundary up to the distance of Earth, while there is a strong deformation of the cross section due to the interaction with the structured ambient solar wind. It reveals that the force-free configuration for magnetic clouds seems to be conserved very well, at least in an average sense since we average over the whole cross-section.

Results gained from these MHD simulations turned out to reflect quite well the picture for magnetic clouds which has been derived from long-term in-situ observations. For direct comparison, Fig. 18 presents measurement profiles which are obtained by a virtual spacecraft located near the equatorial plane at $1 \mathrm{AU}$ while the MCs are sweeping over the spacecraft. Profiles of all four propagation scenarios are superposed, and the times of impact are synchronized. Horizontal bars drawn at the top and the bottom of each figure indicate the times during which the spacecraft is located inside the 
MCs. The structures of one-dimensional profiles reveal the same common features. The shock and sheath are clearly visible, which are followed by an expanding MC that is filled with a low- $\beta$ plasma. Major differences regard the magnetic field inside the sheath (whether there is magnetic reconnection or not) and the behavior of plasma in the clouds' wakes.

One big disadvantage of in-situ observations is that they are performed just along a single path, and an additional model is always required to get a more global picture of physical relationships. The global solution obtained from MHD simulations enables a detailed parameterization of the evolution of important quantities in the whole computational domain. This work may serve as a basis under more general conditions in order to support the interpretation of in-situ observations, e.g., in the frame of flux rope fitting techniques (Hidalgo et al., 2000; Mulligan and Russell, 2001; Leitner at al., 2007). Particularly the effects of different magnetic handedness for magnetic clouds have been elaborated clearly here. The consequences of magnetic reconnection between the magnetic cloud and the interplanetary magnetic field became evident.

Acknowledgements. The author appreciates financial support on behalf of the projects 06/9690 from the Austrian Research Community and A3-12T63/2007-1 from the Styrian government. Participation at the ISSS8 was made possible due to the travel fellowship of UCLA. Nikolai Erkaev acknowledges support by RFBR grants Nos. 07-05-00135 and 09-05-91000-ANF. Charles Farrugia received NASA grants NNG06GD41G and NNX08AD11G. Christian Möstl and Ute Amerstorfer work under FWF projects P20145N16 and P21051-N16 of the Austrian Science Foundation, respectively.

Topical Editor R. Forsyth thanks W. Manchester and S. Dasso for their help in evaluating this paper.

\section{References}

Bame, S. J., Asbridge, J. R., Feldman, W. C., Gosling, J. T., and Zwickl, R. D.: Bi-directional streaming of solar wind electrons greater than $80 \mathrm{eV}$ - ISEE evidence for a closed-field structure within the driver gas of an interplanetary shock, Geophys. Res. Lett., 8, 173-176, 1981.

Biernat, H. K., Erkaev, N. V., Farrugia, C. J., Vogl, D. F., and Schaffenberger, W.: MHD effects of the solar wind flow around planets, Nonlin. Processes Geophys., 7, 201-210, doi:10.5194/npg7-201-2000, 2000.

Bittencourt, J. A.: Fundamentals of plasma physics, 3rd ed., Springer, New York, 2004.

Borrini, G., Gosling, J. T., Bame, S. J., and Feldman, W. C.: Helium abundance enhancements in the solar wind, J. Geophys. Res., 87, 7370-7378, 1982.

Bothmer, V. and Schwenn, R.: The structure and origin of magnetic clouds in the solar wind, Ann. Geophys., 16, 1-24, doi:10.5194/angeo-16-1-1998, 1998.

Brio, M. and Wu, C. C.: An upwind differencing scheme for the equations of ideal magnetohydrodynamics, J. Comput. Phys., 75, 400-422, 1988.
Burlaga, L., Sittler, E., Mariani, F., and Schwenn, R.: Magnetic loop behind an interplanetary shock - Voyager, Helios, and IMP8 observations, J. Geophys. Res., 86, 6673-6684, 1981.

Burlaga, L. F.: Magnetic clouds and force-free fields with constant alpha, J. Geophys. Res., 93, 7217-7224, 1988.

Burton, M. E., Sicoe, G. L., and Smith, E. J.: Shapes of strong shock fronts propagating through the coronal streamer belt, J. Geophys. Res., 97, 12283-12286, 1992.

Cargill, P. J., Schmidt, J., Spicer, D. S., and Zalesak, S. T.: Magnetic structure of overexpanding coronal mass ejections: Numerical models, J. Geophys. Res., 105, 7509-7520, 2000.

Cargill, P. J. and Schmidt, J. M.: Modelling interplanetary CMEs using magnetohydrodynamic simulations, Ann. Geophys., 20, 879-890, doi:10.5194/angeo-20-879-2002, 2002.

Dasso, S., Mandrini, C. H., Démoulin, P., and Luoni, M. L.: A new model-independent method to compute magnetic helicity in magnetic clouds, Astron. Astrophys., 455, 349-359, 2006.

Dasso, S., Nakwacki, M. S., Démoulin, P., and Mandrini, C. H.: Progressive transformation of a flux rope to an ICME. Comparative analysis using the direct and fitted expansion methods, Solar Phys., 244, 115-137, 2007.

Dasso, S., Mandrini, C. H., Schmieder, B., Cremades, H., Cid, C., Cerrato, Y., Saiz, E., Démoulin, P., Zhukov, A. N., Rodriguez, L., Aran, A., Menvielle, M., and Poedts, S.: Linking two consecutive nonmerging magnetic clouds with their solar sources, J. Geophys. Res., 114, A02109, doi:10.1029/2008JA013102, 2009.

Démoulin, P. and Dasso, S.: Magnetic cloud models with bent and oblate cross-section boundaries, Astron. Astrophys., 507, 969980, 2009.

Dungey, J. W.: Interplanetary magnetic field and the auroral zones, Phys. Rev. Lett., 6, 47-48, 1961.

Erkaev, N. V., Farrugia, C. J., Biernat, H. K., Burlaga, L. F., and Bachmaier, G. A.: Ideal MHD flow behind interplanetary shocks driven by magnetic clouds, J. Geophys. Res., 100, 19919-19932, 1995.

Erkaev, N. V., Semenov, V. S., and Biernat, H. K.: Two-dimensional MHD model of the reconnection diffusion region, Nonlin. Processes Geophys., 9, 131-138, doi:10.5194/npg-9-131-2002, 2002.

Farrugia, C. J., Richardson, I. G., Burlaga, L. F., Lepping, R. P., and Osherovich, V. A.: Simultaneous observations of solar MeV particles in a magnetic cloud and in the Earths northern tail lobe Implications for the global field line topology of magnetic clouds and for the entry of solar particles into the magnetosphere during cloud passage, J. Geophys. Res., 98, 15497-15507, 1993.

Farrugia, C. J., Fitzenreiter, R. J., Burlaga, L. F., Erkaev, N. V., Osherovich, V. A., Biernat, H. K., and Fazakerley, A.: Observations in the sheath region ahead of a magnetic cloud and in the dayside magnetosheath during magnetic cloud passage, Adv. Space Res., 14, 105-110, 1994.

Farrugia, C. J., Burlaga, L. F., and Lepping, R. P.: Magnetic clouds and the quiet-storm effect at Earth, in: Magnetic Storms, Geophys. Monogr. Ser., 98, 91-106, 1997.

Farrugia, C. J., Vasquez, B., Richardson, I. G., Torbert, R. B., Burlaga, L. F., Biernat, H. K., Mühlbachler, S., Ogilvie, K. W., Lepping, R. P., Scudder, J. D., Berdichevsky, D. E., Semenov, V. S., Kubyshkin, I. V., Phan, T.-D., and Lin, R. P.: A reconnection layer associated with a magnetic cloud, Adv. Space Res., 28, 759-764, 2001. 
Gazis, P. R., Barnes, A., Mihalov, J. D., and Lazarus, A. J.: Solar wind velocity and temperature in the outer heliosphere, J. Geophys. Res., 99, 6561-6573, 1994.

Godunov, S. K.: A finite difference method for the computation of discontinuous solutions of the equations of fluid dynamics, Mat. Sb., 47, 357-393, 1959.

Gosling, J. T., Pizzo, V., and Bame, S. J.: Anomalously low proton temperatures in the solar wind following interplanetary shock waves - evidence for magnetic bottles?, J. Geophys. Res., 78, 2001-2009, 1973.

Gosling, J. T., Baker, D. N., Bame, S. J., Feldman, W. C., Zwickl, R. D., and Smith, E. J.: Bidirectional solar wind electron heat flux events, J. Geophys. Res., 92, 8519-8535, 1987.

Gosling, J. T.: Coronal mass ejections and magnetic flux ropes in interplanetary space, in: Physics of magnetic flux ropes, AGU Washington D.C., 343-364, 1990.

Gosling, J. T., Bame, J., McComas, D. J., Phillips, J. L., Scime, E. E., Pizzo, V. J., Goldstein, B. E., and Balogh, A.: A forwardreverse shock pair in the solar wind driven by over-expansion of a coronal mass ejection: Ulysses observations, Geophys. Res. Lett., 21, 237-240, 1994.

Gosling, J. T., Riley, P., McComas, D. J., and Pizzo, V. J.: Overexpanding coronal mass ejections at high heliographic latitudes observations and simulations, J. Geophys. Res., 103, 1941-1954, 1998.

Gulisano, A. M., Démoulin, P., Dasso, S., Ruiz, M. E., and Marsch, E.: Global and local expansion of magnetic clouds in the inner heliosphere, Astron. Astrophys., 509, A39, doi:10.1051/00046361/200912375, 2010.

Hau, L.-N. and Sonnerup, B. U. Ö.: Two-dimensional coherent structures in the magnetopause: Recovery of static equilibria from single-spacecraft data, J. Geophys. Res., 104, 6899-6918, 1999.

Hidalgo, M. A., Cid, C., Medina, J., and Viñas, A. F.: A new model for the topology of magnetic clouds in the solar wind, Solar Phys., 194, 165-174, 2000.

Hidalgo, M. A.: A study of the expansion and distortion of the cross section of magnetic clouds in the interplanetary medium, J. Geophys. Res., 108, SSH 4-1, 1320, doi:10.1029/2002JA009818, 2003.

$\mathrm{Hu}$, Q. and Sonnerup, B. U. Ö.: Reconstruction of magnetic clouds in the solar wind: Orientations and configurations, J. Geophys. Res., 107, SSH 101, doi:10.1029/2001JA000293, 2002.

Kahler, S. W. and Reames, D. V.: Probing the magnetic topologies of magnetic clouds by means of solar energetic particles, J. Geophys. Res., 96, 9419-9424, 1991.

Klein, L. W. and Burlaga, L. F.: Interplanetary magnetic clouds at 1 AU, J. Geophys. Res., 87, 613-624, 1982.

Leamon, R. J., Smith, C. W., and Ness, N. F.: Characteristics of magnetic fluctuations within coronal mass ejections: The January 1997 event, Geophys. Res. Lett., 25, 2505-2508, 1998.

Leitner, M., Farrugia, C. J., Möstl, C., Ogilvie, K. W., Galvin, A. B., Schwenn, R., and Biernat, H. K.: Consequences of the forcefree model of magnetic clouds for their heliospheric evolution, $\mathrm{J}$. Geophys. Res., 112, A06113, doi:10.1029/2006JA011940, 2007.

Lepping, R. P., Burlaga, L. F., and Jones, J. A.: Magnetic field structure of interplanetary magnetic clouds at 1 AU, J. Geophys. Res., 95, 11957-11965, 1990.

Lepping, R. P., Narock, T. W., and Wu, C.-C.: A scheme for finding the front boundary of an interplanetary magnetic cloud, Ann. Geophys., 27, 1295-1311, doi:10.5194/angeo-27-12952009, 2009.

LeVeque, R. J.: Finite volume methods for hyperbolic problems, Cambridge University Press, 2002.

Liu, Y., Richardson, J. D., and Belcher, J. W.: A statistical study of the properties of interplanetary coronal mass ejections from 0.3 to 5.4 AU, Planet. Space Sci., 53, 3-17, 2005.

Liu, Y., Richardson, J. D., Belcher, J. W., Kasper, J. C., and Elliott, H. A.: Thermodynamic structure of collision-dominated expanding plasma: Heating of interplanetary coronal mass ejections, J. Geophys. Res., 111, A01102, doi:10.1029/2005JA011329, 2006.

Liu, Y., Luhmann, J. G., Huttunen, K. E. J., Lin, R. P., Bale, S. D., Russell, C. T., and Galvin, A. B.: Reconstruction of the 2007 May 22 magnetic cloud: How much can we trust the flux-rope geometry of CMEs?, Astrophys. J., 677, L133-L136, 2008a.

Liu, Y., Manchester, W. B., Richardson, J. D., Luhmann, J. G., Lin, R. P., and Bale, S. D.: Deflection flows ahead of ICMEs as an indicator of curvature and geoeffectiveness, J. Geophys. Res., 113, A00B03, doi:10.1029/2007JA012996, 2008b.

Lopez, R. E.: Solar cycle invariance in solar wind proton temperature relationships, J. Geophys. Res., 92, 11189-11194, 1987.

Low, B. C.: Solar activity and the corona, Solar Phys., 167, $217-$ 265, 1996.

Lugaz, N., Manchester IV, W. B., Roussev, I. I., Tóth, G., and Gombosi, T. I.: Numerical investigation of the homologous coronal mass ejection events from active region 9236, Astrophys. J., 659, 788-800, 2007.

Lugaz, N., Manchester IV, W. B., Roussev, I. I., and Gombosi, T. I.: Observational evidence of CMEs interacting in the inner heliosphere as inferred from MHD simulations, J. Atmos. Solar-Terr. Phys., 70, 598-604, 2008.

Lundquist, S.: Magneto-hydrostatic fields, Ark. Fys., 2, 361-365, 1950.

Manchester, W. B., Gombosi, T. I., Roussev, I., Ridley, A., De Zeeuw, D. L., Sokolov, I. V., Powell, K. G., and Tóth, G.: Modeling a space weather event from the Sun to the Earth: CME generation and interplanetary propagation, J. Geophys. Res., 109, A02107, doi:10.1029/2003JA010150, 2004.

Manchester IV, W. B., Gombosi, T. I., De Zeeuw, D. L., Sokolov, I. V., Roussev, I. I., Powell, K. G., Kóta, J., Tóth, G., and Zurbuchen, T. H.: Coronal mass ejection shock and sheath structures relevant to particle acceleration, Astrophys. J., 622, 1225-1239, 2005.

Manchester IV, W. B., Vourlidas, A., Tóth, G., Lugaz, N., Roussev, I. I., Sokolov, I. V., Gombosi, T. I., De Zeeuw, D. L., and Opher, M.: Three-dimensional MHD simulation of the 2003 October 28 coronal mass ejection: comparison with LASCO coronagraph observations, Astrophys. J., 684, 1448-1460, 2008.

Möstl, C., Miklenic, C., Farrugia, C. J., Temmer, M., Veronig, A., Galvin, A. B., Vršnak, B., and Biernat, H. K.: Two-spacecraft reconstruction of a magnetic cloud and comparison to its solar source, Ann. Geophys., 26, 3139-3152, doi:10.5194/angeo-263139-2008, 2008.

Möstl, C., Farrugia, C. J., Biernat, H. K., Leitner, M., Kilpua, E. K. J., Galvin, A. B., and Luhmann, J. G.: Optimized GradShafranov reconstruction of a magnetic cloud using STEREO-Wind observations, Solar Phys., 256, 427-441, 2009.

Mulligan, T. and Russell, C. T.: Multispacecraft modeling of 
the flux rope structure of interplanetary coronal mass ejections: cylindrically symmetric versus nonsymmmetric topologies, J. Geophys. Res., 106, 10581-10596, 2001.

Newkirk Jr., G., Hundhausen, A. J., and Pizzo, V.: Solar cycle modulation of galactic cosmic rays - speculation on the role of coronal transients, J. Geophys. Res., 86, 5387-5396, 1981.

Odstrčil, D., Dyer, M., and Smith, Z.: Propagation of an interplanetary shock along the heliospheric plasma sheet, J. Geophys. Res., 101, 19973-19986, 1996.

Odstrčil, D. and Pizzo, V. J.: Three-dimensional propagation of CMEs in a structured solar wind flow: 1. CME launched within the streamer belt, J. Geophys. Res., 104, 483-492, 1999.

Odstrcil, D., Linker, J. A., Lionello, R., Mikic, Z., Riley, P., Pizzo, V. J., and Luhmann, J. G.: Merging of coronal and heliospheric numerical two-dimensional MHD models, J. Geophys. Res., 107, SSH 14-1, 1493, doi:10.1029/2002JA009334, 2002.

Odstrcil, D., Riley, P., and Zhao, X. P.: Numerical simulation of the 12 May 1997 interplanetary CME event, J. Geophys. Res., 109, A02116, doi:10.1029/2003JA010135, 2004.

Owens, M. J., Cargill, P. J., Pagle, C., Siscoe, G. L., and Crooker, N. U.: Characteristic magnetic field and speed properties of interplanetary coronal mass ejections and their sheath regions, J. Geophys. Res., 110, A01105, doi:10.1029/2004JA010814, 2005.

Owens, M. J.: Magnetic cloud distortion resulting from propagation through a structured solar wind: Models and observations, J. Geophys. Res., 111, A12109, doi:10.1029/2006JA011903, 2006.

Parker, E. N.: Dynamics of the interplanetary gas and magnetic fields, Astrophys. J., 128, 664-676, 1958.

Parker, E. N.: Interplanetary dynamical processes, New York, Interscience Publishers, 1963.

Paularena, K. I., Zastenker, G. N., Lazarus, A. J., and Dalin, P. A.: Solar wind plasma correlations between IMP 8, INTERBALL-1, and WIND, J. Geophys. Res., 103, 14601-14618, 1998.

Pneuman, G. W. and Kopp, R. A.: Gas-magnetic field interactions in the solar corona, Sol. Phys., 18, 258-270, 1971.

Powell, K. G.: An approximate Riemann solver for magnetohydrodynamics (that works in more than one dimension), ICASE report no. 94-24, NASA Langley Research Center, 1994.

Powell, K. G., Roe, P. L., Linde, T. J., Gombosi, T. I., and de Zeeuw, D. L.: A solution-adaptive upwind scheme for ideal magnetohydrodynamics, J. Comput. Phys., 154, 284-309, 1999.

Richardson, I. G. and Cane, H. V.: Regions of abnormally low proton temperature in the solar wind (1965-1991) and their association with ejecta, J. Geophys. Res., 100, 23397-23412, 1995.

Richardson, J. D., Paularena, K. I., Lazarus, A. J., and Belcher, J. W.: Radial evolution of the solar wind from IMP 8 and Voyager 2, Geophys. Res. Lett., 22, 325-328, 1995.

Richardson, J. D. and Smith, C. W.: The radial temperature profile of the solar wind, Geophys. Res. Lett., 30, 1206, doi:10.1029/2002GL016551, 2003.

Richardson, I. G. and Cane, H. V.: The fraction of interplanetary coronal mass ejections that are magnetic clouds: Evidence for a solar cycle variation, Geophys. Res. Lett., 31, L18804, doi:10.1029/2004GL020958, 2004.

Riley, P. and Crooker, N. U.: Kinematic treatment of coronal mass ejection evolution in the solar wind, Astrophys. J., 600, 10351042, 2004.

Roe, P. L.: Approximate Riemann solvers, parameter vectors, and difference schemes, J. Comput. Phys., 43, 357-372, 1981.
Roussev, I. I., Gombosi, T. I., Sokolov, I. V., Velli, M., Manchester IV, W., DeZeeuw, D. L., Liewer, P., Tóth, G., and Luhmann, J.: A three-dimensional model of the solar wind incorporating solar magnetogram observations, Astrophys. J., 595, L57-L61, 2003.

Schmidt, J. M. and Cargill, P. J.: Magnetic reconnection between a magnetic cloud and the solar wind magnetic field, J. Geophys. Res., 108, SSH 5-1, 1023, doi:10.1029/2002JA009325, 2003.

Schwenn, R. and Marsch, E.: Physics of the inner heliosphere. 1. Large-scale phenomena, Phys. Chem. Space, 20, 1990.

Siscoe, G. and Odstrcil, D.: Ways in which ICME sheaths differ from magnetosheaths, J. Geophys. Res., 113, A00B07, doi:10.1029/2008JA013142, 2008.

Toro, E. F.: Riemann solvers and numerical methods for fluid dynamics, Springer - Berlin Heidelberg, 1999.

Tóth, G., Sokolov, I. V., Gombosi, T. I., Chesney, D. R., Clauer, C. R., De Zeeuw, D. L., Hansen, K. C., Kane, K. J., Manchester, W. B., Oehmke, R. C., Powell, K. G., Ridley, A. J., Roussev, I. I., Stout, Q. F., Volberg, O., Wolf, R. A., Sazykin, S., Chan, A., Yu, B., and Kóta, J.: Space weather modeling framework: A new tool for the space science community, J. Geophys. Res., 110, A12226, doi:10.1029/2005JA011126, 2005.

Tóth, G., De Zeeuw, D. L., Gombosi, T. I., Manchester, W. B., Ridley, A. J., Sokolov, I. V., and Roussev, I. I.: Sun-tothermosphere simulation of the 28-30 October 2003 storm with the Space Weather Modeling Framework, Space Weather, 5, S06003, doi:10.1029/2006SW000272, 2007.

Totten, T. L., Freeman, J. W., and Arya, S.: An empirical determination of the polytropic index for the free-streaming solar wind using Helios 1 data, J. Geophys. Res., 100, 13-17, 1995.

Uralova, S. V. and Uralov, A. M.: WKB approach to the problem of MHD shock propagation through the heliospheric current sheet, Solar Phys., 152, 457-479, 1994.

Vandas, M., Fisher, S., and Geranios, A.: Spherical and cylindrical models of magnetized plasma clouds and their comparison with spacecraft data, Planet. Space Sci., 39, 1147-1154, 1991.

Vandas, M., Fisher, S., Pelant, P., and Geranios, A.: Evidence for spheroidal structure of magnetic clouds, J. Geophys. Res., 98, 21061-21069, 1993.

Vandas, M., Fischer, S., Dryer, M., Smith, Z., and Detman, T.: Simulation of magnetic cloud propagation in the inner heliosphere in two-dimensions. 1: A loop perpendicular to the ecliptic plane, J. Geophys. Res., 100, 12285-12292, 1995.

Vandas, M., Fischer, S., Dryer, M., Smith, Z., and Detman, T.: Simulation of magnetic cloud propagation in the inner heliosphere in two dimensions 2. A loop parallel to the ecliptic plane and the role of helicity, J. Geophys. Res., 101, 2505-2510, 1996.

Wang, C. and Richardson, J. D.: Interplanetary coronal mass ejections observed by Voyager 2 between 1 and 30 AU, J. Geophys. Res., 109, A06104, doi:10.1029/2004JA010379, 2004.

Wang, C., Du, D., and Richardson, J. D.: Characteristics of the interplanetary coronal mass ejections in the heliosphere between 0.3 and 5.4 AU, J. Geophys. Res., 110, A10107, doi:10.1029/2005JA011198, 2005.

Winterhalter, D., Smith, E. J., Burton, M. E., Murphy, N., and McComas, D. J.: The heliospheric plasma sheet, J. Geophys. Res., 99, 6667-6680, 1994.

Zachary, A. L. and Colella, P.: A higher-order Godunov method for the equations of ideal magnetohydrodynamics, J. Comput. Phys., 99, 341-347, 1992. 